\title{
Energy Drinks and Sports Performance, Cardiovascular Risk, and Genetic Associations; Future Prospects
}

\author{
Jorge Gutiérrez-Hellín ${ }^{1}$ (D) and David Varillas-Delgado ${ }^{2, *(D)}$ \\ 1 Exercise and Sport Sciences, Faculty of Health Sciences, Universidad Francisco de Vitoria, \\ 28223 Madrid, Spain; jorge.gutierrez@ufv.es \\ 2 Research Unit, Faculty of Medicine, Universidad Francisco de Vitoria, Pozuelo de Alarcon, \\ 28223 Madrid, Spain \\ * Correspondence: david.varillas@ufv.es; Tel.: +34-91-709-1400 (ext. 1965)
}

check for updates

Citation: Gutiérrez-Hellín, J.; Varillas-Delgado, D. Energy Drinks and Sports Performance, Cardiovascular Risk, and Genetic Associations; Future Prospects. Nutrients 2021, 13, 715. https:// doi.org/10.3390/nu13030715

Academic Editor: James King

Received: 12 January 2021

Accepted: 20 February 2021

Published: 24 February 2021

Publisher's Note: MDPI stays neutral with regard to jurisdictional claims in published maps and institutional affiliations.

Copyright: (c) 2021 by the authors. Licensee MDPI, Basel, Switzerland. This article is an open access article distributed under the terms and conditions of the Creative Commons Attribution (CC BY) license (https:// creativecommons.org/licenses/by/ $4.0 /)$.

\begin{abstract}
The consumption of energy drinks (e.g., containing caffeine and taurine) has increased over the last decade among adolescents and athletes to enhance their cognitive level and improve intellectual and athletic performance. Numerous studies have shown that drinking moderate doses of such drinks produces beneficial effects, as they considerably boost the sporting performance of elite athletes in various sports, including both endurance and explosive events. However, apart from their ergogenic effects, the regular consumption of energy drinks also increases blood pressure and consequently incites problems such as hypertension, tachycardia, and nervousness, all of which can lead to cardiovascular disorders. A potential positive correlation between genetics and the moderate consumption of energy drinks and athletic performance has recently been reported; notwithstanding, a better understanding of the genetic variants involved in metabolism is a key area for future research to optimize the dose of energy drink consumed and obtain the maximal ergogenic effect in elite sports. The aim of this literature review, therefore, is to present the results of recent studies, classifying them according to the differences in the associations between energy drinks and: (i) Athletic performance; (ii) cardiovascular risk factors while practicing sports; and (iii) genetic associations and future prospects between the consumption of energy drinks and performance.
\end{abstract}

Keywords: energy drinks; sports performance; caffeine; taurine; health risk; genetics

\section{Introduction}

Energy drinks first made their appearance in Europe and Asia in 1960. Energy drinks first appeared in Austria in 1987 with a well-known brand and erupted across the globe over the following years. Their consumption has increased exponentially as they have gained in popularity and it has now become a multibillion-dollar industry [1].

It is necessary to differentiate between energy drinks and traditional beverages (coffee, tea, isotonic, hypotonic and hypertonic sports drinks, and soft drinks such as cola). Energy drinks have a high caffeine content which is normally combined with large amounts of vitamins, minerals, taurine, amino acids, and different mixtures of phytochemicals [2].

This type of drink has gained particular prominence, as evidenced by its consumption by various demographic groups, with and without risks of disease, such as youths, workers, students, professional athletes, amateur athletes, and nightlife revelers [3]. No countries restrict or place age limits on the consumption and sale of energy drinks, so they are readily accessible to all populations and ages.

Regarding physical exercise, energy drinks form part of training prioritization in terms of the physical qualities to condition, nutritional practices, pharmacological approach or psychological techniques that can improve training adaptations and/or the output of the exercise [4]. This includes aids that may benefit individuals when exercising, increase the efficiency of the exercise and/or improve subsequent recovery $[4,5]$. 
The use of dietary supplements is widespread throughout the general population, but it takes on particular importance for those who practice sports and their consumption by athletes corresponds to a significant proportion of their sales [4,6-8]. Dietary supplements can play an important role in helping athletes achieve an ideal intake of calories and nutrients. However, they should never be considered as a substitute for a healthy diet [4].

Energy drinks have emerged as a key dietary supplement to enhance athletic performance, particularly in the acute consumption, with the effects of caffeine and taurine being the most studied in several different sports [9-11]. The association between performance and the consumption of energy drinks has been demonstrated in American football and soccer [12-14], athletics [15-17], volleyball [18], and handball [19], amongst others. Although there is comprehensive evidence of the positive association between the consumption of these drinks and improved sporting performance, there are risks in terms of the potential for cardiovascular problems due to hypertension, altered sleep patterns in adolescents, aggravation of mental illnesses, physiological dependence and an increased possibility of subsequent addiction [20,21], while their potential for toxicity can result in tachycardia, arrhythmia, vomiting, convulsions and even death [22]. The adverse effects of energy drink intake may occur in healthy people, but some people may be particularly prone to complications. High-risk groups include young, caffeine-deficient or caffeine-sensitive pregnant women, competitive athletes, and people with underlying cardiovascular disease [23]. Moreover, the effects of chronic high-dose caffeine and taurine intake in children, adolescents and athletes are not yet known [22].

Possibly one of the most interesting areas for research regarding the consumption of energy drinks associated with athletic performance and the observed cardiovascular risks would be to study the genetic markers that indicate a greater predisposition to improve performance by consuming energy drinks [24] and a protective effect against the damage they can cause to the cardiovascular system. The field of nutrigenomics is expanding our understanding of sports performance [25], but it is still a long way from obtaining evidence-based knowledge. In the area of caffeine, recent evidence related to cytochrome P450 1A2 (CYP1A2) -163C>A polymorphism has helped optimize the caffeine dose an athlete needs to improve their performance [26-28] in endurance sports [29], as well as in team sports and explosive efforts $[19,28,30,31]$.

Several studies have shown significant relationships between the consumption of energy drinks, sports performance and increased prevalence of cardiovascular risk factors. However, the results reported to date remain inconsistent, so a full general description of the studies in this field is necessary. For a more detailed analysis of the matter, we have classified recent studies according to the differences in the associations between the energy drinks and: (i) Athletic performance; (ii) cardiovascular risk factors while practicing sports; and (iii) genetic associations and future prospects between the consumption of energy drinks and performance.

\section{Energy Drinks and Sport Performance}

\subsection{Caffeine and Sport Performance}

Energy drinks are beverages that combine different substances among their ingredients, including vitamins, minerals, taurine, amino acids, and mixtures of phytochemicals such as caffeine [2]. Their main impacts on sporting performance are attributed to the effects of caffeine and taurine.

Caffeine (1,3,7-trimethylxanthine) is a phytochemical found in the leaves, fruits, and seeds of various plants such as coffee, tea, and mate [32]. It is a socially-acceptable drug that has been used as an ergogenic aid or performance enhancer in multiple sporting disciplines for many years now. It can be used in various physical forms as a legal nutritional method of improving sports performance in training sessions and competition [33]. Its consumption was banned by the World Anti-Doping Agency (WADA) up until 2004. Athletes were sanctioned if they presented urinary caffeine concentrations above $12 \mu \mathrm{gm} / \mathrm{L}$. From 2004, caffeine was removed from the list of substances and methods prohibited in- 
and out-of-competition and placed on the monitoring program [34]. Since then, its sale and consumption has remained unrestricted in the context of international sports.

Caffeine can be consumed in different forms. It is available in gels, bars, chewing gums, lozenges, coffee, tea, cocoa products, cola drinks, and energy drinks. The delivery form determines the rate at which it is absorbed and reaches the bloodstream [35]. The fastest means of caffeine absorption is in the form of chewing gum [36] due to drugs absorbed via the buccal cavity bypass intestinal and hepatic first pass metabolism, which potentially increases their extent of absorption of caffeinated substances. Of all the caffeine consumption routes, the most common method of ingesting caffeine across the globe is in energy drinks [37].

Several researchers have tried to determine the optimal doses necessary to enhance sports performance by studying caffeine dose-response relationships and the subsequent ergogenic effect on athletic performance. In this regard, a randomized study by Pasman et al. [38] analyzed the ergogenic dose-response effect during endurance training in nine well-trained cyclists and observed a significant effect at doses of $5 \mathrm{mg} / \mathrm{kg}$ or more (endurance times of $47 \pm 13 \mathrm{~min}, 58 \pm 11 \mathrm{~min}, 59 \pm 12 \mathrm{~min}$ and $58 \pm 12 \mathrm{~min}$ for $0 \mathrm{mg}, 5 \mathrm{mg}$, $9 \mathrm{mg}$ and $13 \mathrm{mg}$ caffeine $/ \mathrm{kg}$ of body weight, respectively $[p<0.05])$. In a controlled trial, Polito et al. [39] studied the acute effects of ingesting two different doses of caffeine ( 3 and $6 \mathrm{mg} / \mathrm{kg}$ ) on performance during a resistance training session (chest press, shoulder press and biceps curl exercises) compared to placebo. The results showed that the placebo group completed significantly fewer repetitions $(93.6 \pm 22.4)$ than the groups that took $3 \mathrm{mg} / \mathrm{kg}$ $(108.0 \pm 19.9, p=0.02)$ and $6 \mathrm{mg} / \mathrm{kg}(109.3 \pm 19.8, p=0.03)$ of caffeine, while there were no differences between the two caffeine doses. Another controlled study, published by Jenkins et al. [40], compared the dose-response effect of 1,2 and $3 \mathrm{mg} / \mathrm{kg}$ on the athletic performance of 13 trained cyclists against placebo. The results revealed an increase in performance of $4 \%$ after ingesting $2 \mathrm{mg} / \mathrm{kg}$ and $3 \%$ with $3 \mathrm{mg} / \mathrm{kg}$ upon completing a 15 -min time trail at $60 \%$ of $\mathrm{VO}_{2}$ max.

The literature also includes studies in which the acute consumption of at least $3 \mathrm{mg} / \mathrm{kg}$ of caffeine was necessary to improve the performance in different disciplines and movements, as well as cognitively [41]. The optimal dose range to obtain ergogenic effects is 3-6 mg/ $\mathrm{kg}$, whether taken acutely in capsules or consumed as an energy drink [16]. Doses of 6 and $9 \mathrm{mg} / \mathrm{kg}$ had the same effects without producing an accumulative dose-response effect [42], while the total doses ingested in energy drinks were around 40-325 mg of caffeine, which is comparable to doses of $3-6 \mathrm{mg} / \mathrm{kg}$ in capsules [9].

The ergogenic effects of caffeine during exercise were first recorded in the scientific community over 100 years ago [43]. Caffeine is known to have a positive effect by increasing physical performance in endurance sports [44], intermittent exercises such as team and racket sports [45,46], and high-intensity disciplines (from 1-60 min) such as swimming, rowing and middle- and long-distance running [33].

\subsection{Taurine and Sport Performance}

Taurine (2-aminoethanesulphonic acid) is a stimulant commonly found in energy drinks. It is the most prevalent free amino acid found in mammalian muscle tissue [47] and is present in high concentrations in meat and seafood [48].

Taurine corresponds to $50-60 \%$ of free amino acids in mammals and fulfils some essential biological functions. Within muscle fiber, taurine stimulates the release of $\mathrm{Ca}^{2+}$ from the sarcoplasmic reticulum and maintains the sensitivity of contractile elements to $\mathrm{Ca}^{2+}$, acting directly on excitation-contraction-relaxation processes, contractile properties and force production [49], and also exhibiting positive effects on athletic performance in animal models [50]. These findings have been confirmed in both cardiac and skeletal myocytes [51], with such effects considered responsible for the improved performance reported in some studies, such as that of Balshaw et al. [52] who demonstrated the positive effect of the acute consumption of $1000 \mathrm{mg}$ of taurine on sports performance in a $3 \mathrm{~km}$ time trial test. A randomized study by Rutherford et al. [53] also observed a 16\% increase in 
fat oxidation during a cycle ergometer test at a moderate intensity $\left(66.5 \pm 1.9 \% \mathrm{VO}_{2} \max \right)$ for $90 \mathrm{~min}$ in trained cyclists. While another trial attributed prescribed an antioxidant effect to taurine that facilitated the equilibrium in the mitochondrial matrix and improved the efficiency of energy fluctuations in the form of ATP in mice muscle fibers (treatment decreased muscle taurine levels to $<40 \%$ of controls; $p<0.05$ ) [50].

The amounts of taurine in energy drinks range between 71 and $3105 \mathrm{mg}$ [9]. However, some studies have used higher doses (4000-6000 mg/day for 7 days) [54], with the most frequently used doses to date ranging between 1000 and $2000 \mathrm{mg}$, according to the literature [55].

\subsection{Energy Drinks Combining Caffeine and Taurine in Sport Performance}

Combining caffeine with taurine enhances its effects on the sarcoplasmic reticulum in cardiomyocytes. Both taurine and caffeine have shown in vitro physiological effects on intracellular calcium concentrations in vascular smooth muscle. The highest levels of both substances are found in the heart tissues of patients with congestive heart failure and experimental models of cardiac hypertrophy [56], and can also produce a positive inotropic effect, supporting the idea that caffeine and taurine may act in synergy [56].

The consumption of energy drinks serves different purposes depending on the population drinking them. One of their main uses is to enhance sports performance [57]. This, together with their increased consumption due to their popularity and worldwide availability, has to lead to an increase in the number of scientific studies published in recent years. Various clinical trials have shown that drinking an energy drink that combines caffeine and taurine correlates positively with an improved athletic performance in a range of sporting situations. A study by Quinlivan et al. [58] compared the effect of an energy drink (Red Bull $\left.{ }^{\circledR}\right), 3 \mathrm{mg} / \mathrm{kg}$ of caffeine and placebo on the sporting performance of cyclists through $1 \mathrm{~h}$ time trial at 75\% peak power output and found that the group who consumed the energy drink improved their performance by $120 \pm 172 \mathrm{~s}(3768 \pm 257 \mathrm{~s})(3.1 \%, p=0.043)$ compared to placebo (3877 $\pm 260 \mathrm{~s})$ and the caffeine group $(3757 \pm 278 \mathrm{~s})$ by $109 \pm 153 \mathrm{~s}$ $(2.8 \%, p=0.039)$. Similarly, a double-blind, placebo experimented study presented by Cureton et al. [59] in 16 trained cyclists reported that subjects completed 15-23\% more work during a 135 min continuous ride after consuming a caffeinated sports drink with taurine compared to placebo $(p<0.05)$. A controlled trial performed by Ivy et al. [60] in trained cyclists who consumed an energy drink (Red Bull $\left.{ }^{\circledR}\right)$ found that the athletes completed a $1 \mathrm{~h}$ time trial at 70\% maximum Watts (Wmax) in a shorter time than for placebo (3690 $\pm 64 \mathrm{~s}$ vs. $3874 \pm 93 \mathrm{~s}, p<0.01)$; which are very similar results to those of Kovacs and Cureton studies $[59,61]$.

In other sporting disciplines with predominantly cyclic and acyclic movements such as football, a randomized study by Lara et al. [13] found that $60 \mathrm{~min}$ after drinking an energy drink (Fure ${ }^{\circledR}$ : Standardized as $3 \mathrm{mg} / \mathrm{kg}$ of body weight, maltodextrin $(300 \mathrm{mg} / \mathrm{g})$, taurine (400 mg/g), L-carnitine (40 mg/g), B-group vitamins $(10 \mathrm{mg} / \mathrm{g}$ ) and sodium bicarbonate $(100 \mathrm{mg} / \mathrm{g}))$, the athletes' performance improved compared to placebo in countermovement jumps (heights of $27.4 \pm 3.8 \mathrm{~cm}$ vs. $26.6 \pm 4.0 \mathrm{~cm} ; p<0.05$ ), a $7 \times 30 \mathrm{~m}$ sprint test (top speeds of $24.5 \pm 1.7 \mathrm{~km} / \mathrm{h}$ vs. $24.2 \pm 1.6 \mathrm{~km} / \mathrm{h} ; p<0.05$ ) and a simulated football match monitored with GPS devices (total distances run of $7.087 \pm 1.501 \mathrm{~m}$ vs. $6.631 \pm 1.618 \mathrm{~m} ; p<0.05$ ).

In repeated high-intensity efforts, Del Coso et al. [62] also described that the consumption of an energy drink (Fure ${ }^{\circledR}$ ) increased the athletic performance of elite rugby sevens players as evidenced by greater muscle power output during a $15 \mathrm{~s}$ maximal jump test ( $25.6 \pm 11.8$ vs. $23.5 \pm 10.1 \mathrm{~kW}$ with and without the energy drink; $p<0.05)$, running pace during matches $(95.4 \pm 12.7$ vs. $87.5 \pm 8.3 \mathrm{~m} / \mathrm{min}, p<0.05)$ and pace at sprint velocity $(6.1 \pm 3.4$ vs. $4.6 \pm 3.3 \mathrm{~m} / \mathrm{min}, p<0.05)$. In another study, Del Coso et al. [63] also showed the effect of Fure ${ }^{\circledR}$ on athletic performance in sports requiring accelerations and repeated high-intensity movements (elite field hockey players). In this case, the energy drink reduced the distance covered at moderate-intensity running $(793 \pm 135$ and $712 \pm 116$ m-placebo drink vs. energy drink; $p=0.03$ ) and increased the distance covered 
at high-intensity running ( $303 \pm 67$ vs. $358 \pm 117 \mathrm{~m} ; p=0.05)$ and sprinting ( $85 \pm 41$ vs. $117 \pm 55 \mathrm{~m} ; p=0.02)$.

A positive correlation between energy drink consumption and improved sporting performance has also been observed in sports such as volleyball. Pérez-López et al. [64] reported a positive correlation between athletic performance and drinking Fure ${ }^{\circledR}$. Compared to placebo, the energy drink increased ball velocity in the standing spike $(19.2 \pm 2.1 \mathrm{vs}$. $19.7 \pm 1.9 \mathrm{~m} / \mathrm{s} ; p=0.023)$ and jumping spike $(17.9 \pm 2.2 \mathrm{vs} .18 .8 \pm 2.2 \mathrm{~m} / \mathrm{s} ; p=0.038)$ and also improved jump height in the squat jump $(28.1 \pm 3.2 \mathrm{vs} .29 .4 \pm 3.6 \mathrm{~cm} ; p=0.028)$, CMJ (countermovement jump) ( $32.0 \pm 4.6$ vs. $33.1 \pm 4.5 \mathrm{~cm} ; p=0.018)$, spike jump ( $43.3 \pm 4.7$ vs. $44.4 \pm 5.0 \mathrm{~cm} ; p=0.025)$ and block jump ( $35.2 \pm 5.1 \mathrm{vs.} 36.1 \pm 5.1 \mathrm{~cm} ; p=0.044)$.

Another randomized study in a sample of sprint swimmers published by Lara et al. [65] revealed a similar effect to that observed in previous works. Consuming Fure ${ }^{\circledR}$ improved the CMJ ( $49.4 \pm 5.3$ vs. $50.9 \pm 5.2 \mathrm{~cm} ; p<0.05)$, maximal force during the handgrip test with the right hand $(481 \pm 49$ vs. $498 \pm 43 \mathrm{~N} ; p<0.05)$ and peak power output $(273 \pm 55$ vs. $303 \pm 49 \mathrm{~W} ; p<0.05$ ), while reducing the time needed to complete a simulated $50 \mathrm{~m}$ competitive swim $(27.8 \pm 3.4$ vs. $27.5 \pm 3.2 \mathrm{~s} ; p<0.05)$.

Furthermore, the effect of energy drink intake has been studied in racket sports such as badminton and tennis. Abian et al. [66] evidenced an ergogenic effect of Fure ${ }^{\circledR}$ consumption on athletic performance in 15 elite badminton players as they increased their squat jump (SJ) height (34.5 \pm 4.7 vs. $36.4 \pm 4.3 \mathrm{~cm} ; p<0.05)$, squat jump peak power $(p<0.05)$, CMJ $(37.7 \pm 4.5$ vs. $39.5 \pm 5.1 \mathrm{~cm} ; p<0.05)$ and $\mathrm{CMJ}$ peak power $(p<0.05)$ compared to placebo. They also observed more total impacts during a simulated badminton match in comparison with placebo (7395 \pm 1594 vs. $7707 \pm 2033$ impacts; $p<0.05)$. Gallo-Salazar et al. [67] conducted a randomized study that also found a positive correlation between the consumption of energy drinks containing caffeine and taurine and improved athletic performance in elite youth tennis players. Drinking Fure ${ }^{\circledR}$ increased handgrip strength by $4.2 \pm 7.2 \%$ $(p=0.03)$ in both hands, high intensity running pace $(46.7 \pm 28.5 \mathrm{vs} .63 .3 \pm 27.7 \mathrm{~m} / \mathrm{h}$; $p=0.02)$, and number of sprints $(12.1 \pm 1.7$ vs. $13.2 \pm 1.7 ; p=0.05)$ during the simulated match. Compared to the placebo drink, subjects tended to produce a greater maximal running velocity during the sprint test $(22.3 \pm 2.0 \mathrm{vs} .22 .9 \pm 2.1 \mathrm{~km} / \mathrm{h} ; p=0.07)$ and win more points on serve $(49.7 \pm 9.8 \%$ vs. $56.4 \pm 10.0 \% ; p=0.07)$ with the caffeinated energy drink. Considering the increased performance in power-specific movements, there is a proven positive correlation between the consumption of energy drinks and improved performance in strength exercises, endurance work, vertical jump tests and specific sprint tests or accelerations (Table 1).

When assessing the effect of consuming energy drinks with caffeine and taurine on the physical component of athletic performance, randomized, double-blind studies show that such consumption correlates positively with aerobic endurance capacity. This was evidenced in treadmill tests conducted by Rahnama et al. [68], who found that $\mathrm{VO}_{2}$ max was greater in athletes who ingested energy drinks $(p<0.05)$, as well as in cycle ergometer tests by Ganio et al. [69], in which a group taking caffeine, taurine and B-complex vitamins solution presented improved physical performance between minutes 30 and 120 of the exercise $(p<0.001)$. With respect to the basic physical quality of strength, Sünram-Lea et al. [70] have also shown that the use of $330 \mathrm{~mL}$ of energy drinks correlates positively with improvements in isometric strength exercises. Ratamess et al. [71] studied dynamic strength in squat and bench press exercises in subjects given a combination with caffeine, taurine, creatine and essential branched-chain amino acids (BCAAs), reporting statistically significant improvements $(p<0.05)$. In a study by Astley et al. [72], after consuming an energy drink with $64 \mathrm{mg}$ of caffeine and $800 \mathrm{mg}$ of taurine per $200 \mathrm{~mL}, 15$ resistance-trained athletes performed better in specific strength tests compared to placebo, as measured by the number of repetitions in a unilateral knee extension test with the dominant leg $(9.5 \pm 0.8$ vs. $11.5 \pm 0.9$ reps; $p=0.001)$ and bench presses $(8.1 \pm 0.5$ vs. $10.2 \pm 0.4$ reps; $p=0.01)$.

Alford et al. [73] have also shown that drinking an energy drink (Red Bull $\left.{ }^{\circledR}\right)$ presents a positive association with athletic performance, reporting significantly $(p<0.05)$ improved 
aerobic endurance (sustained max. heart rate of $65-75 \%$ ) and anaerobic performance (sustained max. speed) on cycle ergometers.

In a recent randomized study, Chtourou et al. [74] found that the acute effects of consuming an energy drink included reductions in reaction times, depression, confusion, fatigue, anger, anxiety, rating of perceived exertion (RPE) and affective load scores compared to placebo in 19 physical education students.

However, focusing on jumping movements, results reported in the literature reflect a lesser effect. For instance, the results presented in the Jacobson et al. [75] study, suggest that there is more evidence and a stronger positive association for this type of drink in upper body muscle groups than in large, complex muscle bundles in the lower body.

However, although we have described different studies which demonstrate a positive correlation between the consumption of energy drinks and athletic performance, it is worth highlighting that some studies did not report this positive association (Table 1). A recent study by Thomas et al. [76] investigated the effects of energy drinks on the sporting performance of eSports players (before and after playing three rounds of League of Legends with $15 \mathrm{~min}$ recovery between rounds) by assessing attention (Erikson Flanker Test), reaction speed (Go/No-Go test) and working memory (n-back test); only the memory test revealed a statistically significant association $(p=0.004)$, while physical components such as handgrip strength and finger tap speed presented a negative association with sporting performance $(p=0.803)$. Similarly, Umaña-Alvarado and Moncada-Jiménez [77] did not observe a positive effect on physical performance in terms of endurance in 11 runners who competed in two $10 \mathrm{~km}$ races, as there were no significant differences between their times when they drank the energy drink or placebo $(p>0.05)$. Candow et al. [78] did not find any differences between an energy drink and placebo among 17 university students after performing a time-to-exhaustion treadmill run at $80 \% \mathrm{VO}_{2} \max \left(\operatorname{Red} \mathrm{Bull}^{\circledR}\right.$ : $12.6 \pm 3.8$ min, placebo: $11.8 \pm 3.4 \mathrm{~min}$; perceived exertion Red Bull ${ }^{\circledR}: 17.1 \pm 2.0$, placebo: $16.6 \pm 1.8$ ). Nor did Dall'Agnol and Souza [79] report any differences during an incremental exercise test performed in 22 healthy volunteers after they ingested either $160 \mathrm{mg}$ of caffeine with $2000 \mathrm{mg}$ of taurine or placebo (332.50 \pm 56.83 vs. $342 \pm 40.60 \mathrm{~W})$, while Kammerer et al. [80] did not find any differences in $\mathrm{VO}_{2}$ max values after volunteer soldiers consumed an energy drink compared to a placebo-controlled scenario. In the same vein, Pettitt et al. [81] observed that consuming an energy drink did not improve the gas exchange threshold in eight recreationally trained subjects during a graded exercise test on a cycle ergometer. Nelson et al. [82] also reported that there was no significant difference in the time-to-exhaustion between placebo and energy drink trials ( $43.8 \pm 9.3$ vs. $45.5 \pm 9.8 \mathrm{~min}$; $p=0.62$ ).

With respect to anaerobic factors, we found three studies that did not evidence a positive association between the consumption of an energy drink and peak anaerobic power $(p>0.05)$ in strength and power athletes, namely those by Hoffman et al. [83], Gwacham and Wagner [12] and Eckerson et al. [84]. Regarding the basic physical quality of muscle strength, a randomized, double-blind study by Goel et al. [85] did not discover any significant differences in maximal voluntary contraction between an energy drink and control (males: Before and after consumption of energy drink $381 \pm 37$ vs. $371 \pm 36 \mathrm{~N}$ and control drink $375 \pm 61$ vs. $363 \pm 36 \mathrm{~N}$; females: Energy drink $227 \pm 23$ vs. $227 \pm 32 \mathrm{~N}$ and control $234 \pm 46$ vs. $228 \pm 37 \mathrm{~N}$ ). All detailed results concerning the correlation between energy drinks and sport performance are presented in Table 1. 
Table 1. Characteristics of studies referring to the association between energy drinks and sport performance.

\begin{tabular}{|c|c|c|c|c|c|c|c|c|}
\hline \multicolumn{9}{|c|}{ Statistically Significant Positive Association } \\
\hline First Author/Country/ & Year & Study Design & Sample (n) & Gender/Age/Mean Age & Caffeine Dosage & Taurine Dosage & Exercise Performance Test & Result \\
\hline $\begin{array}{l}\text { Kovacs et at. / the } \\
\text { Netherlands [61] }\end{array}$ & 1998 & $\begin{array}{l}\text { Randomized double } \\
\text { blind, placebo } \\
\text { controlled, crossover }\end{array}$ & $\begin{array}{c}15 \\
\begin{array}{c}\text { (Well-trained triathletes } \\
\text { and cyclists) }\end{array}\end{array}$ & Male, $23.3 \pm 0.9 y$ & $\begin{array}{l}\text { Drinks of } 14 \mathrm{~mL} / \mathrm{kg} \\
\text { BM of a placebo and } \\
\text { four carbohydrate- } \\
\text { electrolyte solution } \\
\text { containing } 150 \mathrm{mg} / \mathrm{L} \\
\mathrm{CAF}, 225 \mathrm{mg} / \mathrm{L} \mathrm{CAF} \\
\text { and } 320 \mathrm{mg} / \mathrm{L} \mathrm{CAF}\end{array}$ & $\begin{array}{l}\text { All drinks contained } 70 \\
\mathrm{mg} / \mathrm{L}\end{array}$ & $\begin{array}{l}\text { Warm-up protocol }(20 \mathrm{~min}) \\
\text { and a 1-h time trial cycling } \\
\text { performance }\end{array}$ & $\begin{array}{c}\text { Improved in min with } \\
\text { caffeine supplementation: } \\
62.5 \pm 1.3,61.5 \pm 1.1, \\
60.4 \pm 1.0,58.9 \pm 1.0 \text { and } \\
58.9 \pm 1.2 \mathrm{~min} \text { for placebo- } \\
\text { carbohydrate-electrolyte } \\
\text { solution, } \\
\text { carbohydrate-electrolyte } \\
\text { solution-150mg/L, } \\
\text { carbohydrate-electrolyte } \\
\text { solution-225mg/L, and } \\
\text { carbohydrate-electrolyte } \\
\text { solution-320mg/L, } \\
\text { respectively }(p<0.05)\end{array}$ \\
\hline $\begin{array}{l}\text { Alford et al./the UK } \\
{[\text { [73] }}\end{array}$ & 2001 & $\begin{array}{l}\text { Double-blind, } \\
\text { randomized, repeated } \\
\text { measures }\end{array}$ & $\begin{array}{c}39 \\
\text { (Healthy volunteers) }\end{array}$ & Both, $18-30$ y & $\begin{array}{l}\text { Red Bull }{ }^{\circledast} \text { Energy } \\
\text { Drink contains } \\
\text { carbonated water, } \\
\text { caffeine }(80 \mathrm{mg})\end{array}$ & $\begin{array}{l}\text { Red Bull Energy Drink } \\
\text { contains taurine (1000 } \\
\text { mg) }\end{array}$ & $\begin{array}{l}\text { Psychomotor performance } \\
\text { (reaction time, } \\
\text { concentration, memory), } \\
\text { subjective alertness and } \\
\text { physical endurance on cycle } \\
\text { ergometer }\end{array}$ & $\begin{array}{c}\text { Red Bull }{ }^{\circledR} \text { Energy Drink } \\
\text { significantly improved } \\
(p<0.05) \text { aerobic endurance } \\
\text { (maintaining } 65-75 \% \text { max. } \\
\text { heart rate) and anaerobic } \\
\text { performance (maintaining } \\
\text { max. speed) }\end{array}$ \\
\hline $\begin{array}{c}\text { Cureton et al./the USA } \\
\text { [59] }\end{array}$ & 2007 & $\begin{array}{l}\text { A double-blind, } \\
\text { placebo-controlled, } \\
\text { repeated-measures } \\
\text { experimental design }\end{array}$ & $\begin{array}{c}16 \\
\text { (Trained cyclist) }\end{array}$ & Male, $27 \pm 7 y$ & $\begin{array}{c}\text { Commercially available } \\
7 \% \\
\text { CHO-electrolyte sports } \\
\text { drink containing } 195 \\
\text { mg/L caffeine }\end{array}$ & $1.92 \mathrm{~g} / \mathrm{L}$ taurine & $\begin{array}{l}\text { Cycled continuously for a } \\
\text { total of } 135 \text { min, alternating } \\
\text { the exercise intensity } \\
\text { between } 60 \% \text { and } 75 \% \\
\mathrm{VO}_{2} \text { max every } 15 \text { min for } \\
\text { the first } 120 \text { min. The last } 15 \\
\text { min of cycling, the subjects } \\
\text { were instructed to ride as } \\
\text { hard as possible }\end{array}$ & $\begin{array}{l}\text { The performance ride was } \\
15-23 \% \text { greater for energy } \\
\text { drink compared to placebo. } \\
\text { Ratings of perceived } \\
\text { exertion were lower with } \\
\text { energy drink. } \\
\text { Strength loss was less for } \\
\text { energy drink than for the } \\
\text { other beverages or placebo } \\
\text { (5\% vs. } 15 \%) \text {. }\end{array}$ \\
\hline $\begin{array}{c}\text { Ratamess et al./the } \\
\text { USA [71] }\end{array}$ & 2007 & $\begin{array}{l}\text { Randomized, } \\
\text { double-blind crossover }\end{array}$ & $\begin{array}{c}8 \\
\text { (Resistance trained) }\end{array}$ & Male, $20 \pm 2$ y & $\begin{array}{c}\text { Amino Shooter, } \\
\text { Champion Nutrition, } \\
\text { Concord with } 110 \mathrm{mg} \\
\text { of caffeine }\end{array}$ & $1500 \mathrm{mg} / \mathrm{L}$ taurine & $\begin{array}{c}5 \text { min of light stationary } \\
\text { cycling at a self-selected } \\
\text { cadence and an additional } \\
\text { component of very light } \\
\text { stretching and performance } \\
\text { of } 2-3 \text { light to moderate sets } \\
\text { of the squat. Protocol was of } \\
6 \text { sets of the squat exercise } \\
\text { with a load equivalent to } \\
75 \% \text { of subjects } \\
\text { predetermined 1-RM. }\end{array}$ & $\begin{array}{c}\text { Area under the curve of } \\
\text { resistance-exercise volume } \\
\text { was significantly less in } \\
\text { baseline than energy drink } \\
(10 \%) \text { and placebo (8.6\%). } \\
\text { Energy drink } \\
(18.4 \% \pm 12.0 \%) \text { was } \\
\text { significantly lower for } \\
\text { fatigue rate than baseline } \\
(32.9 \% \pm 8.4 \%)\end{array}$ \\
\hline Ivy et al./the USA [60] & 2009 & $\begin{array}{l}\text { Double-blind, } \\
\text { randomized and } \\
\text { placebo-controlled }\end{array}$ & $\begin{array}{c}12 \\
\text { (Cyclist athletes) }\end{array}$ & Both, $27 \pm 3 y$ & $\begin{array}{l}\text { Red Bull Energy Drink } \\
\text { contains carbonated } \\
\text { water, caffeine }(80 \mathrm{mg})\end{array}$ & $\begin{array}{l}\text { Red Bull Energy Drink } \\
\text { contains taurine (1000 } \\
\text { mg) }\end{array}$ & $\begin{array}{l}\text { Trained cyclists consumed } \\
500 \mathrm{~mL} \text { of either flavoured } \\
\text { placebo or Red Bull }{ }^{\circledR} \text { Energy } \\
\text { Drink. Performance was } \\
\text { measured to } 1 \mathrm{hr} \text { of cycling } \\
\text { at } 70 \% \text { Wmax }\end{array}$ & $\begin{array}{l}\text { Performance improved with } \\
\text { energy drink compared } \\
\text { with placebo }(3.690 \pm 64 \mathrm{~s} \\
\text { vs. } 3, .874 \pm 93 \mathrm{~s}, p<0.01)\end{array}$ \\
\hline
\end{tabular}


Table 1. Cont.

\begin{tabular}{|c|c|c|c|c|c|c|c|c|}
\hline \multicolumn{9}{|c|}{ Statistically Significant Positive Association } \\
\hline First Author/Country/ & Year & Study Design & Sample (n) & Gender/Age/Mean Age & Caffeine Dosage & Taurine Dosage & Exercise Performance Test & Result \\
\hline $\begin{array}{c}\text { Ganio et al./the USA } \\
\text { [69] }\end{array}$ & 2010 & $\begin{array}{l}\text { Double-blind, } \\
\text { randomized, crossover, } \\
\text { repeated measures }\end{array}$ & $\begin{array}{c}15 \\
\text { (Cyclist) }\end{array}$ & Male, $27 \pm 6 y$ & $125 \mathrm{mg} / \mathrm{L}$ of caffeine & $1920 \mathrm{mg} / \mathrm{L}$ of L-taurine & $\begin{array}{l}14 \text { male cyclists cycled for } \\
120 \text { min submaximal and } \\
\text { then completed a } 15-\text { min } \\
\text { performance trial. Also, } \\
\text { maximal voluntary leg } \\
\text { isometric extension }\end{array}$ & $\begin{array}{c}\text { Total work accumulated } \\
\text { during performance trial } \\
\text { was greater }(p<0.05) \text { in } \\
\text { energy drink }(233 \pm 34 \mathrm{KJ}) \\
\text { than placebo }(205 \pm 52 \mathrm{KJ}) \\
\text { but not in carbohydrate- } \\
\text { electrolyte-only solution } \\
(225 \pm 39 \mathrm{KJ}) \text { vs. placebo. } \\
\text { MVC ( }) \text { declined } \\
\text { ( } p<0.001) \text { from pre to post } \\
\text { in placebo ( } 988 \pm 213 \mathrm{KJ} \text { to } \\
851 \pm 191 \mathrm{KJ}) \text { and } \\
\text { carbohydrate-electrolyte- } \\
\text { only solution }(970 \pm 172 \mathrm{KJ} \\
\text { to } 870 \pm 163 \mathrm{KJ}) \text { but not in } \\
\text { energy drink }(953 \pm 171 \mathrm{KJ} \\
\text { to } 904 \pm 208 \mathrm{KJ}) \text {. At Minutes } \\
60,90,105, \text { and } 120 \mathrm{RPE} \text { was } \\
\text { lower in energy drink than } \\
\text { in placebo }(p<0.001) \text {. }\end{array}$ \\
\hline $\begin{array}{c}\text { Rahnama et al./Iran } \\
{[68]}\end{array}$ & 2010 & $\begin{array}{l}\text { Randomized, placebo } \\
\text { controlled, } \\
\text { counterbalanced } \\
\text { double-blind }\end{array}$ & $\begin{array}{c}10 \\
\text { (Student athletes) }\end{array}$ & Male, $22 \pm 2$ y & $\begin{array}{l}\text { Red Bull( }{ }^{\circledR} \text { Energy } \\
\text { Drink caffeine ( } 80 \text { mg) }\end{array}$ & $\begin{array}{l}\text { Red Bull }{ }^{\circledR} \text { Energy } \\
\text { Drink } 1000 \mathrm{mg} \text { of } \\
\text { taurine }\end{array}$ & $\begin{array}{l}\text { Maximal oxygen } \\
\text { consumption tests on a } \\
\text { treadmill. }\end{array}$ & $\begin{array}{c}\text { Greater value in } \mathrm{VO}_{2} \max \\
\text { and time to exhaustion for } \\
\text { the Red Bull } \\
\text { trial comp Hype } \\
\text { tred to placebo } \\
(p<0.05)\end{array}$ \\
\hline
\end{tabular}


Table 1. Cont.

\begin{tabular}{|c|c|c|c|c|c|c|c|c|}
\hline \multicolumn{9}{|c|}{ Statistically Significant Positive Association } \\
\hline First Author/Country/ & Year & Study Design & Sample (n) & Gender/Age/Mean Age & Caffeine Dosage & Taurine Dosage & Exercise Performance Test & Result \\
\hline $\begin{array}{l}\text { Del Coso et al./Spain } \\
\text { [62] }\end{array}$ & 2013 & $\begin{array}{l}\text { Double-blind, placebo } \\
\text { controlled and } \\
\text { randomized } \\
\text { experimental }\end{array}$ & $\begin{array}{c}16 \\
\text { (Rugby-seven athletes) }\end{array}$ & Female, $23 \pm 2$ y & $\begin{array}{c}\text { (Fure }^{\circledR} \text {, ProEnergetics, } \\
\text { Spain) provide a dose } \\
\text { of } 3 \mathrm{mg} \text { of caffeine per } \\
\mathrm{kg} \text { of BM. }\end{array}$ & $18.7 \mathrm{mg} / \mathrm{kg}$ & $\begin{array}{c}\text { Participants performed } 15 \mathrm{~s} \\
\text { maximal jumps test, } \\
6 \times 30 \mathrm{~m} \text { sprint test and } 3 \\
\text { rugby matches (running } \\
\text { distance) }\end{array}$ & $\begin{array}{c}\text { Fure }{ }^{\circledR} \text { increased the ball } \\
\text { velocity }(19.2 \pm 2.1 \mathrm{~m} / \mathrm{s} \text { vs } \\
19.7 \pm 1.9 \mathrm{~m} / \mathrm{s}, p=0.023), \\
\text { jumping spike } \\
(17.9 \pm 2.2 \mathrm{~m} / \mathrm{s} \text { vs. } \\
18.8 \pm 2.2 \mathrm{~m} / \mathrm{s}, p=0.038) \\
\text { and jump height in the SJ } \\
(28.1 \pm 3.2 \mathrm{~cm} \text { vs. } \\
29.4 \pm 3.6 \mathrm{~cm}, p=0.028), \\
\mathrm{CMJ}(32.0 \pm 4.6 \mathrm{vs} . \\
33.1 \pm 4.5 \mathrm{~cm}, p=0.018), \\
\text { spike jump }(43.3 \pm 4.7 \mathrm{~cm} \\
\text { vs. } 44.4 \pm 5.0 \mathrm{~cm}, p=0.025), \\
\text { and block jump }(35.2 \pm 5.1 \\
\text { cm vs. } 36.1 \pm 5.1 \mathrm{~cm}, \\
p=0.044) .\end{array}$ \\
\hline Lara et al./Spain [13] & 2014 & $\begin{array}{l}\text { Double-blind, placebo } \\
\text { controlled, randomized } \\
\text { experimental design }\end{array}$ & $\begin{array}{c}18 \\
\text { (Soccer players) }\end{array}$ & Female, $21 \pm 2 \mathrm{y}$ & $\begin{array}{l}\text { (Fure }{ }^{\circledR}, \text { ProEnergetics, } \\
\text { Spain) }\end{array}$ & $18.7 \mathrm{mg} / \mathrm{kg}$ & $\begin{array}{l}\text { Standardized warm-up and } \\
\text { CMJ, } 7 \times 30 \mathrm{~m} \text { sprint and } \\
\quad 2 \times 40 \mathrm{~m}\end{array}$ & $\begin{array}{c}\text { Fure }^{\circledR} \text { increased } \\
\text { performance in CMJ } \\
(26.6 \pm 4.0 \mathrm{~cm} \text { vs } \\
27.4 \pm 3.8 \mathrm{~cm} ; p<0.05), \\
7 \times 30 \mathrm{~m} \text { sprint }(24.2 \pm 1.6 \\
\mathrm{km} / \mathrm{h} \text { vs. } 24.5 \pm 1.7 \\
\mathrm{~km} / \mathrm{h} ; p<0.05) \text { and in } \\
\text { match simulation } 2 \times 40 \mathrm{~m} \\
(6.631 \pm 1.618 \mathrm{~m} \text { with the } \\
\text { placebo drink and } \\
7.087 \pm 1.501 \mathrm{~m} \text { with the } \\
\text { caffeinated energy drink } \\
(p<0.05) .\end{array}$ \\
\hline $\begin{array}{l}\text { Del Coso et al./Spain } \\
\text { [18] }\end{array}$ & 2014 & $\begin{array}{l}\text { Double-blind, placebo } \\
\text { controlled, randomized } \\
\text { experimental }\end{array}$ & $\begin{array}{c}15 \\
\text { (Volleyball players) }\end{array}$ & Male, $22 \pm 7$ y & $\begin{array}{c}\text { (Fure }^{\circledR}, \text { ProEnergetics, } \\
\text { Spain) }\end{array}$ & $18.7 \mathrm{mg} / \mathrm{kg}$ & $\begin{array}{l}\text { Volleyball-specific tests: } \\
\text { standing spike test, maximal } \\
\text { SJ, maximal CMJ, 15RJ test, } \\
\text { and agility T-test }\end{array}$ & $\begin{array}{c}\text { Energy drink increased the } \\
\text { spike test }(73 \pm 9 \mathrm{~km} / \mathrm{h} \\
75 \pm 10 \mathrm{~km} / \mathrm{h}, p<0.05) \text { and } \\
\text { jump height in S } \\
(31.1 \pm 4.3 \mathrm{~cm} \text { vs. } \\
32.7 \pm 4.2 \mathrm{~cm}, p<0.05), \mathrm{CMJ} \\
(35.9 \pm 4.6 \mathrm{vs} .37 .7 \pm 4.4 \mathrm{~cm} \\
p<0.05), \text { and } 15 \mathrm{RJ} \\
(29.0 \pm 4.0 \mathrm{~cm} \text { vs. } \\
30.5 \pm 4.6 \mathrm{~cm}, p<0.05) \text {. The } \\
\text { agility test time was } \\
\text { significantly reduced with } \\
\text { the caffeinated energy drink } \\
(10.8 \pm 0.7 \mathrm{~s} \text { vs. } 10.3 \pm 0.4 \mathrm{~s}, \\
p<0.05) . \\
\text { Players performed } \\
\text { successful volleyball actions } \\
\text { more frequently } \\
(24.6 \% \pm 14.3 \% \text { vs } \\
34.3 \% \pm 16.5 \%, p<0.05) \\
\text { compared to placebo. }\end{array}$ \\
\hline
\end{tabular}


Table 1. Cont.

\begin{tabular}{|c|c|c|c|c|c|c|c|c|}
\hline \multicolumn{9}{|c|}{ Statistically Significant Positive Association } \\
\hline First Author/Country/ & Year & Study Design & Sample (n) & Gender/Age/Mean Age & Caffeine Dosage & Taurine Dosage & Exercise Performance Test & Result \\
\hline Abian et al./Spain [66] & 2015 & $\begin{array}{l}\text { Double-blind, placebo } \\
\text { controlled and } \\
\text { randomized } \\
\text { experimental design }\end{array}$ & $\begin{array}{l}15 \\
\text { (Elite badminton } \\
\text { players) }\end{array}$ & Male, $25 \pm 7 y$ & $\begin{array}{c}\text { (Fure }{ }^{\circledR}, \text { ProEnergetics, } \\
\text { Spain) }\end{array}$ & $18.7 \mathrm{mg} / \mathrm{kg}$ & $\begin{array}{c}\text { Handgrip maximal force } \\
\text { production, smash jump } \\
\text { without and with } \\
\text { shuttlecock, SJ, CMJ and the } \\
\text { agility } t \text {-test. } \\
\text { 45-min simulated } \\
\text { badminton match was } \\
\text { played. }\end{array}$ & $\begin{array}{c}\text { Energy drink increased SJ } \\
\text { height }(34.5 \pm 4.7 \mathrm{~cm} \text { vs. } \\
36.4 \pm 4.3 \mathrm{~cm} ; p<0.05), \text { SJ } \\
\text { peak power }(p<0.05) \text {, CMJ } \\
(37.7 \pm 4.5 \mathrm{vs} .39 .5 \pm 5 \mathrm{~cm} \text {; } \\
p<0.05) \text { and CMJ peak } \\
\text { power }(p<0.05) \text {. } \\
\text { An increased number of } \\
\text { total impacts was found } \\
\text { during the badminton } \\
\text { match }(7395 \pm 1594 \text { impacts } \\
\text { vs. } 7707 \pm 2033 \text { impacts, } \\
p<0.05) .\end{array}$ \\
\hline $\begin{array}{l}\text { Gallo-Salazar } \\
\text { et al./Spain [67] }\end{array}$ & 2015 & $\begin{array}{l}\text { Double-blind, placebo } \\
\text { controlled and } \\
\text { randomized } \\
\text { experimental }\end{array}$ & $\begin{array}{c}14 \\
\text { (Young elite-level } \\
\text { tennis players) }\end{array}$ & Male $16 \pm 1 \mathrm{y}$ & $\begin{array}{c}\text { (Fure }{ }^{\circledR}, \text { ProEnergetics, } \\
\text { Spain) }\end{array}$ & $18.7 \mathrm{mg} / \mathrm{kg}$ & $\begin{array}{c}\text { Handgrip-strength test, a } \\
\text { maximal-velocity serving } \\
\text { test, and an } 8 \times 15 \text {-m sprint } \\
\text { test. } \\
\text { Were carried out simulated } \\
\text { singles match (best of } \\
3 \text { sets). }\end{array}$ & $\begin{array}{l}\text { Energy drink increased } \\
\text { handgrip force by } \\
4.2 \% \pm 7.2 \%(p=0.03) \text { in } \\
\text { both hands, the running } \\
\text { pace at high intensity } \\
(46.7 \pm 28.5 \mathrm{vs} .63 .3 \pm 27.7 \\
\mathrm{km} / \mathrm{h}, p=0.02), \text { and the } \\
\text { number of prints } \\
(12.1 \pm 1.7 \mathrm{vs} .13 .2 \pm 1.7 \\
p=0.05) \text { during the } \\
\text { simulated match. }\end{array}$ \\
\hline
\end{tabular}


Table 1. Cont.

\begin{tabular}{|c|c|c|c|c|c|c|c|c|}
\hline \multicolumn{9}{|c|}{ Statistically Significant Positive Association } \\
\hline First Author/Country/ & Year & Study Design & Sample (n) & Gender/Age/Mean Age & Caffeine Dosage & Taurine Dosage & Exercise Performance Test & Result \\
\hline $\begin{array}{l}\text { Pérez-López } \\
\text { et al./Spain [64] }\end{array}$ & 2015 & $\begin{array}{l}\text { Double-blind, placebo } \\
\text { controlled and } \\
\text { randomized } \\
\text { experimental }\end{array}$ & $\begin{array}{c}13 \\
\text { (Elite volleyball) }\end{array}$ & Female, $25 \pm 5 \mathrm{y}$ & $\begin{array}{c}\text { (Fure }{ }^{\circledR}, \text { ProEnergetics, } \\
\text { Spain) }\end{array}$ & $18.7 \mathrm{mg} / \mathrm{kg}$ & $\begin{array}{l}\text { Standardized heating and } \\
\text { performed standing spike, } \\
\text { jumping spike, spike jump, } \\
\text { blocking jump, SJ, CMJ, } \\
\text { manual dynamometry, and } \\
\text { agility } t \text {-test. }\end{array}$ & $\begin{array}{c}\text { Energy drink increased the } \\
\text { ball velocity }(19.2 \pm 2.1 \mathrm{~m} / \mathrm{s} \\
\text { vs. } 19.7 \pm 1.9 \mathrm{~m} / \mathrm{s}, \\
p=0.023), \text { jumping spike } \\
\text { (17.9 } \pm 2.2 \mathrm{~m} / \mathrm{s} \text { vs. } \\
18.8 \pm 2.2 \mathrm{~m} / \mathrm{s}, p=0.038) \\
\text { and jump height in the SJ } \\
(28.1 \pm 3.2 \mathrm{~cm} \text { vs. } \\
29.4 \pm 3.6 \mathrm{~cm}, p=0.028), \\
\text { CMJ }(32.0 \pm 4.6 \mathrm{~cm} \text { vs. } \\
33.1 \pm 4.5 \mathrm{~cm}, p=0.018) \text {, SJ } \\
(43.3 \pm 4.7 \mathrm{~cm} \text { vs. } \\
44.4 \pm 5.0 \mathrm{~cm} p=0.025) \\
\text { and block jump } \\
(35.2 \pm 5.1 \mathrm{~cm} \text { vs. } \\
36.1 \pm 5.1 \mathrm{~cm}, p=0.044) .\end{array}$ \\
\hline $\begin{array}{l}\text { Quinlivan } \\
\text { et al./Australia [58] }\end{array}$ & 2015 & $\begin{array}{l}\text { Double-blind, } \\
\text { crossover }\end{array}$ & $\begin{array}{c}11 \\
\text { (Cyclist and triathletes) }\end{array}$ & Male, $31.6 \pm 6.1 \mathrm{y}$ & $\begin{array}{c}\text { Red Bull }{ }^{\circledR} \text { Energy } \\
\text { Drink contains caffeine } \\
(80 \mathrm{mg})\end{array}$ & $\begin{array}{c}\text { Red Bull }{ }^{\circledR} \text { Energy } \\
\text { Drink contains taurine } \\
(1000 \mathrm{mg})\end{array}$ & $\begin{array}{l}1 \mathrm{~h} \mathrm{cycling} \text { at } 75 \% \text { peak } \\
\text { power output }\end{array}$ & $\begin{array}{c}\text { Red Bull } \mathrm{l}^{\circledR} \text { intake } \\
\text { significantly increased } \\
\text { sports performance } \\
109 \pm 153 \mathrm{~s}(2.8 \%, p=0.039) \\
\text { in comparison with placebo } \\
120 \pm 172 \mathrm{~s}(3.1 \%, p=0.043) .\end{array}$ \\
\hline $\begin{array}{c}\text { Del Coso et al./Spain } \\
\text { [63] }\end{array}$ & 2016 & $\begin{array}{l}\text { Double-blind, placebo } \\
\text { controlled and } \\
\text { randomized } \\
\text { experimental }\end{array}$ & $\begin{array}{c}13 \\
\text { (Field hockey players) }\end{array}$ & Male, $23 \pm 4$ y & $\begin{array}{c}\text { (Fure }{ }^{\circledR}, \text { ProEnergetics, } \\
\text { Spain) }\end{array}$ & $18.7 \mathrm{mg} / \mathrm{kg}$ & $\begin{array}{c}2 \times 25 \text { min simulated field } \\
\text { hockey game (total distance, } \\
\text { distance high intensity, } \\
\text { distance low intensity and } \\
\text { sprints) }\end{array}$ & $\begin{array}{c}\text { Energy drink reduced the } \\
\text { distance covered at } \\
\text { moderate-intensity running } \\
(793 \pm 135 \text { and } 712 \pm 116, \\
\text { respectively, } p=0.03) \text {, the } \\
\text { distance covered at } \\
\text { high-intensity running } \\
(303 \pm 67 \mathrm{~m} \text { and } 358 \pm 117 \\
\mathrm{m}, p=0.05) \text { and sprinting } \\
(85 \pm 41 \mathrm{~m} \text { and } 117 \pm 55 \mathrm{~m}, \\
\text { respectively, } p=0.02) \text { in } \\
\text { comparison with placebo. }\end{array}$ \\
\hline $\begin{array}{c}\text { Jacobson et al./the USA } \\
\text { [75] }\end{array}$ & 2018 & $\begin{array}{c}\text { Randomized, } \\
\text { double-blind and } \\
\text { placebo-controlled }\end{array}$ & $\begin{array}{c}36 \\
\text { (Healthy volunteers) }\end{array}$ & Both, $23 \pm 2 y$ & $\begin{array}{l}\text { Energy drink with } 240 \\
\text { mg of caffeine }\end{array}$ & $\begin{array}{l}\text { Energy drink with } 200 \\
\text { mg of taurine }\end{array}$ & $\begin{array}{l}3 \text { separate trials of } \mathrm{CMJ} \text { and } \\
\text { isolated forehand stroke } \\
\text { with } 15 \mathrm{~s} \text { rest intervals }\end{array}$ & $\begin{array}{l}\text { The energy drink group } \\
\text { increased a significant } \\
\text { velocity }(p=0.05) \text { and } W \text { for } \\
\text { the forehand stroke, but not } \\
\text { for the CMJ, regarding to } \\
\text { placebo.. }\end{array}$ \\
\hline
\end{tabular}


Table 1. Cont.

\begin{tabular}{|c|c|c|c|c|c|c|c|c|}
\hline \multicolumn{9}{|c|}{ Statistically Significant Positive Association } \\
\hline First Author/Country/ & Year & Study Design & Sample (n) & Gender/Age/Mean Age & Caffeine Dosage & Taurine Dosage & Exercise Performance Test & Result \\
\hline Astley et al./Brazil [72] & 2018 & $\begin{array}{l}\text { Double-blind } \\
\text { cross-over randomized }\end{array}$ & $\begin{array}{c}15 \\
\begin{array}{c}\text { (Resistance-trained } \\
\text { athletes) }\end{array}\end{array}$ & Male, $21 \pm 0.3 y$ & $\begin{array}{l}\text { The energy drink with } \\
\text { soda water containing } \\
\text { caffeine }(64 \mathrm{mg} / 200 \\
\mathrm{mL})\end{array}$ & $\begin{array}{l}\text { The energy drink } \\
\text { containing taurine }(800 \\
\mathrm{mg} / 200 \mathrm{~mL})\end{array}$ & $\begin{array}{c}\text { Maximum repetition test } \\
\text { (80\% 1-RM) in bench press, } \\
\text { unilateral leg extension, } \\
\text { handgrip test, standing long } \\
\text { jump and repeated sprint } \\
\text { ability }\end{array}$ & $\begin{array}{c}\text { Energy Drink intake } \\
\text { increased performance } \\
\text { compared to the placebo for } \\
\text { the number of repetitions in } \\
\text { the unilateral knee } \\
\text { extension test of the } \\
\text { dominant leg } \\
\text { (11.5 } \pm 0.9 \text { reps vs. } \\
9.5 \pm 0.8 \text { reps, } p=0.001) \text { and } \\
\text { bench press }(10.2 \pm 0.4 \text { reps } \\
\text { vs. } 8.1 \pm 0.5 \text { reps, } p=0.01) \text {. } \\
\text { Increased isometric strength } \\
\text { in the hand-grip maximal } \\
\text { test in the right } \\
\text { (53.7 } \pm 1.5 \mathrm{~kg} \text { vs. } \\
47.7 \pm 1.6 \mathrm{~kg}, p=0.02) \text { and } \\
\text { left hand }(52.9 \pm 1.5 \mathrm{~kg} \text { vs. } \\
45.9 \pm 1.3 \mathrm{~kg}, p=0.02) .\end{array}$ \\
\hline $\begin{array}{c}\text { Chtourou et al./France } \\
\text { [74] }\end{array}$ & 2019 & $\begin{array}{l}\text { Randomized double } \\
\text { blind, } \\
\text { placebo-controlled, } \\
\text { counterbalanced and } \\
\text { crossover }\end{array}$ & $\begin{array}{c}19 \\
\text { (Physical-education } \\
\text { students) }\end{array}$ & Male, $21 \pm 1 \mathrm{y}$ & $\begin{array}{l}\text { Red Bull }{ }^{\circledR} \text { Energy } \\
\text { Drink contains } \\
\text { carbonated water, } \\
\text { caffeine }(80 \mathrm{mg})\end{array}$ & $\begin{array}{l}\text { Red Bull }{ }^{\circledR} \text { Energy } \\
\text { Drink contains taurine } \\
\text { (1000 mg) }\end{array}$ & $\begin{array}{l}\text { During } 60 \mathrm{~min} \text {, the subjects } \\
\text { developed visual reaction } \\
\text { time, handgrip test and } 30-\mathrm{s} \\
\text { Wingate }\end{array}$ & $\begin{array}{l}\text { Energy drinks improves } \\
\text { peak and mean power } \\
\text { output, handgrip force, pre- } \\
\text { and post-exercise blood } \\
\text { glucose, blood pressure, and } \\
\text { vigor, correlated with } \\
\text { reduction of fatigue, anxiety } \\
\text { and anger. } \\
\text { Reductions in reaction times, } \\
\text { depression, confusion, } \\
\text { fatigue, anger, anxiety, RPE, } \\
\text { and affective load scores } \\
\text { were observed after energy } \\
\text { drink compared to placebo. } \\
\text { Energy drinks improves } \\
\text { physical performances and } \\
\text { reaction times with RPE, } \\
\text { affective load, and pre- and } \\
\text { post-exercise blood } \\
\text { glucose levels. }\end{array}$ \\
\hline \multicolumn{9}{|c|}{ No statistically significant positive association } \\
\hline $\begin{array}{c}\text { Umaña-Alvarado and } \\
\text { Moncada- } \\
\text { Jiménez/Costa Rica } \\
\text { [77] }\end{array}$ & 2005 & $\begin{array}{l}\text { Double-blind and } \\
\text { randomized crossover }\end{array}$ & $\begin{array}{c}11 \\
\text { (Runners or triathletes) }\end{array}$ & Male, $30 \pm 11 \mathrm{y}$ & $\begin{array}{c}\text { The commercially } \\
\text { available ED provided } \\
\text { (for } 100 \mathrm{~mL}) 32 \mathrm{mg} / \mathrm{mL} \\
\text { of caffeine }\end{array}$ & $\begin{array}{l}\text { The commercially } \\
\text { available ED provided } \\
\text { (for } 100 \mathrm{~mL} \text { ) } 400 \\
\mathrm{mg} / \mathrm{mL} \text { of taurine }\end{array}$ & $\begin{array}{l}\text { Participants completed two } \\
10 \mathrm{~km} \text { cross country run }\end{array}$ & $\begin{array}{l}\text { No significant differences } \\
\text { were found between mean } \\
\text { racing times; however, } \\
\text { ratings of perceived exertion } \\
\text { were significantly lower } \\
\text { when participants ingested } \\
\text { the energy drink vs placebo } \\
(7.02 \pm 1.21 \text { vs. } 8.01 \pm 0.75 \\
p<0.05)\end{array}$ \\
\hline
\end{tabular}


Table 1. Cont.

\begin{tabular}{|c|c|c|c|c|c|c|c|c|}
\hline \multicolumn{9}{|c|}{ Statistically Significant Positive Association } \\
\hline First Author/Country/ & Year & Study Design & Sample (n) & Gender/Age/Mean Age & Caffeine Dosage & Taurine Dosage & Exercise Performance Test & Result \\
\hline $\begin{array}{c}\text { Candow et al./Canada } \\
{[\text { [78] }}\end{array}$ & 2009 & $\begin{array}{l}\text { Double-blind, } \\
\text { crossover, repeated } \\
\text { measures }\end{array}$ & $\begin{array}{c}17 \\
\text { (University students) }\end{array}$ & Both, $21 \pm 4 \mathrm{y}$ & $\begin{array}{l}\text { Sugar-free Red Bull } \\
\text { with } 2 \mathrm{mg} / \mathrm{kg} \text { of } \\
\text { caffeine }\end{array}$ & $\begin{array}{l}\text { Sugar-free Red Bull } \\
\text { with } 25 \mathrm{mg} / \mathrm{kg} \text { of } \\
\text { taurine }\end{array}$ & $\begin{array}{l}\text { Run time-to-exhaustion at } \\
80 \% \mathrm{VO}_{2} \text { max treadmill test }\end{array}$ & $\begin{array}{c}\text { No differences in run } \\
\text { time-to-exhaustion (Red } \\
\text { Bull }{ }^{\circledR}: 12.6 \pm 3.8 \mathrm{~min}, \\
\text { placebo: } 11.8 \pm 3.4 \mathrm{~min} \text { ), } \\
\text { perceived exertion (Red } \\
\text { Bull: } 17.1 \pm 2.0, \text { placebo: } \\
16.6 \pm 1.8) \text {, or blood lactate } \\
\text { between groups }\end{array}$ \\
\hline $\begin{array}{l}\text { Dall'Agnol and } \\
\text { Souza/Brazil [79] }\end{array}$ & 2009 & $\begin{array}{l}\text { Double-blind and } \\
\text { randomized crossover }\end{array}$ & $\begin{array}{c}22 \\
\text { (Healthy volunteers) }\end{array}$ & Male, $26 \pm 4 y$ & $\begin{array}{l}\text { Energy drink with } 160 \\
\text { mg of caffeine }\end{array}$ & $\begin{array}{l}\text { Energy drink with } 2000 \\
\text { mg of taurine }\end{array}$ & $\begin{array}{l}\text { Participants completed and } \\
\text { incremental test on } \\
\text { cycle-ergometer }\end{array}$ & $\begin{array}{c}\text { There was an increase of } 10 \\
\text { W with the administration } \\
\text { of the experimental drink, } \\
\text { without statistical } \\
\text { significance }(342 \pm 40.60 \mathrm{~W} \\
\text { vs. } 332.50 \pm 56.83 \mathrm{~W} \\
p>0.05)\end{array}$ \\
\hline $\begin{array}{l}\text { Hoffman et al./the } \\
\text { USA [83] }\end{array}$ & 2009 & $\begin{array}{l}\text { Randomized } \\
\text { double-blind and } \\
\text { crossover }\end{array}$ & $\begin{array}{l}12 \\
\text { (Strength-power } \\
\text { athletes) }\end{array}$ & Male, $21 \pm 1 \mathrm{y}$ & $\begin{array}{l}\text { Redline Extreme } \\
\text { contained } 158 \mathrm{mg} \text { of } \\
\text { caffeine }\end{array}$ & No taurine & $\begin{array}{l}\text { Reaction test and Wingate } \\
\text { (20 s Wingate anaerobic } \\
\text { power test) }\end{array}$ & $\begin{array}{c}\text { Significant difference in } \\
\text { reaction test was seen } \\
\text { between energy drink and } \\
\text { placebo in both average } \\
\text { number of targets struck } \\
\text { (55.8 } \pm 7.4 \text { vs. } 51.9 \pm 7.4, \\
\text { respectively) and percent of } \\
\text { targets struck (71.9 } \pm 10.5 \% \\
\text { vs. } 66.8 \pm 10.9 \% \text {, } \\
\text { respectively). } \\
\text { No significant differences } \\
\text { between trials were seen in } \\
\text { any anaerobic power } \\
\text { measure. Subjective feelings } \\
\text { of energy (3.5 } \pm 0.5 \text { vs. } \\
3.1 \pm 0.5) \text { and focus } \\
\text { (3.8 } \pm 0.5 \text { vs. } 3.3 \pm 0.7) \text { were } \\
\text { significantly higher during } \\
\text { energy drink compared } \\
\text { to placebo }\end{array}$ \\
\hline $\begin{array}{l}\text { Gwacham and } \\
\text { Wagner/the USA [12] }\end{array}$ & 2012 & $\begin{array}{l}\text { Double-blind, } \\
\text { randomized and } \\
\text { crossover }\end{array}$ & $\begin{array}{c}20 \\
\text { (Football players) }\end{array}$ & Male, $20 \pm 2 y$ & $\begin{array}{l}\text { AdvoCare Spark } \\
\text { energy drink contained } \\
120 \mathrm{mg} \text { of caffeine }\end{array}$ & $\begin{array}{l}\text { AdvoCare Spark } \\
\text { energy drink contained } \\
200 \mathrm{mg} \text { of taurine }\end{array}$ & $\begin{array}{l}\text { Sprint performance and } \\
\text { anaerobic power }\end{array}$ & $\begin{array}{l}\text { Energy drink did not } \\
\text { significantly affect powwer } \\
(3.84, p=0.066) \text { or sprint } \\
\text { time }(3.06, p=0.097) \text {. There } \\
\text { was a significant interaction } \\
\text { effect between caffeine use } \\
\text { and the beverage for sprint } \\
\text { times (4.62, } p=0.045) \text {, as } \\
\text { well as for anaerobic power } \\
(5.40, p=0.032) \text {, indicating a } \\
\text { confounding effect. }\end{array}$ \\
\hline
\end{tabular}

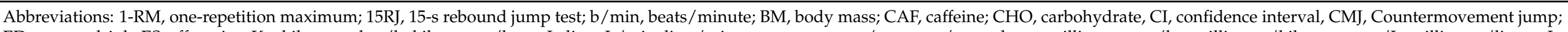

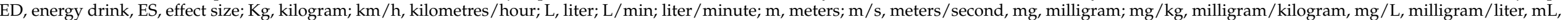

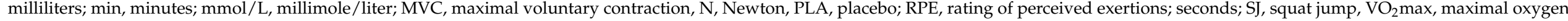
consumption; $\mathrm{W}$, Watts; $\mathrm{y}$, years. 


\section{Energy Drinks and Cardiovascular Risk Factors}

Coffee and caffeine influence the cardiovascular system through their positive inotropic and chronotropic effects, affecting the central nervous system by stimulating locomotor activity and anxiogenic effects. This underlines the need to examine whether these effects may be harmful to health, particularly in the world of sport [86].

Reissig et al. [87] have described several effects linked to excessive caffeine consumption. Over a lifetime, people should only consume large amounts of caffeine for short periods, but this sort of consumption is more common on a regular basis. Furthermore, some people use caffeine to improve their concentration and memory or enhance their physical performance and, in some cases, could develop a dependence syndrome. Caffeine use transforms into "abuse" when individuals develop an uncontrolled need to consume caffeine, even if it is harmful to their health; it transforms into "dependence" when mechanisms of tolerance and abstinence develop, and certain chronic usage habits make caffeine even more damaging. Along with caffeine dependence, subjects who consume extremely high doses continuously for years, ignoring all safety concerns by combining two or more sources of caffeine, for example, coffee and energy drinks, without any evidence that such combinations provide any desirable benefits [86].

Given the significant number of incidents reported among energy drink consumers, it seems pertinent to summarize the available data and establish causal links between the use of these products and the development of health complications. Occasional to moderate consumption of these drinks seems to pose little risk to healthy adults. However, excessive consumption related to their combination with drugs in amounts that far exceed the manufacturers' recommended intakes could induce negative consequences for human health, especially among subjects with cardiovascular disorders [88].

The risk factors may increase the rate of adverse events, particularly cardiovascular events in individuals who consume energy drinks, due to underlying conditions [37], and they may suffer a caffeine overdose, as has been reported in the literature [22,88-91]. A lethal dose of caffeine has been noted as $5 \mathrm{~g}$, which is equivalent to approximately 42 cups of coffee with $120 \mathrm{mg}$ caffeine/cup [90]. Sepkowitz et al. [92] suggested that the acute intake of $3 \mathrm{~g}$ of caffeine can provoke significant side effects, which may even be fatal, with arrhythmia being the most common factor producing death by this lethal dose. A review by Nawrot et al. [93] stated that a healthy adult can consume up to $400 \mathrm{mg}$ of caffeine/day (equivalent to $6 \mathrm{mg} / \mathrm{kg}$ in individuals weighing less than $65 \mathrm{~kg}$ ) without being associated with any adverse effects.

The combined use of caffeine and ephedra has been reported also as a risk factor for cardiovascular problems [94]. There is evidence to suggest that the short-term use of ephedra, with caffeine, promotes short-term weight loss. One example is the metaanalysis by Shekelle et al. [95] in which subjects who took caffeine and ephedrine lost around $0.9 \mathrm{~kg} /$ month more over a short period than the placebo group $(p<0.01)$, no regarding long-term weight loss to support the use of ephedra for athletic performance $(p>0.05)$. Ephedra is known to be ergogenic during anaerobic exercises, such as bench presses $(p<0.05)$, especially when taken with caffeine; however, a point to consider is that systolic blood pressure increased significantly before both tests in subjects treated with ephedrine compared to the other tests [96]. A clinical trial by Haller et al. [97] carried out in 16 healthy subjects showed an increment in the stimulating and metabolic effects of combined ephedrine $(25 \mathrm{mg})$ and caffeine $(200 \mathrm{mg})$ as they increased systolic blood pressure (maximum difference of $11.7 \pm 9.4 \mathrm{mmHg}$ compared to placebo; $p=0.0005$ ) and heart rate (maximum difference of $5.9 \pm 8.8$ beats $/ \mathrm{min} ; p=0.001$ ). The study demonstrated that, individually, ephedrine and caffeine had modest effects, but in combination, they produced significant cardiovascular, metabolic, and hormonal responses at moderate doses, data which should be taken into account to avoid such risks when indicating the dose to produce the desired ergogenic effect. 


\section{Energy Drinks, Sport Performance, and Genetics}

The main enzyme responsible for caffeine metabolism is cytochrome P450 1A2 (CYP1A2), more specifically polymorphism c.-163A >C (rs762551), which corresponds to approximately $95 \%$ of caffeine clearance known to date, being known that caffeine metabolism is also carried out by xanthine oxidase and N-acetyltransferase 2 (NAT2) [86,98].

What is more, polymorphism c.1976T $>C$ (rs5751876) in gene ADORA2A (adenosine A2A receptor) has been shown to modulate sleep-wake activity [99], contribute to individual sensitivity to caffeine's effects on sleep [100], increasing susceptibility to caffeineinduced anxiety [101,102].

In the case of taurine, its effects have been confirmed in animal models. For example, an animal model was used to study the effects of taurine administration on antidepressantlike behaviors in rats and depression-related signal transduction in the hippocampus [103]. Similarly, taurine and $\beta$-alanine supplementation were found to be viable therapeutic strategies to improve the fatigue resistance of dystrophic skeletal muscle in mice [104].

Several studies have shown that the rate of caffeine metabolism may have implications for athletic performance, but findings are still currently ambiguous [29,105-107]. Some publications and certain sports present ergogenic effects that may help improve performance. For example, Guest et al. [29] conducted $10 \mathrm{~km}$ cycling time trials and found that $2 \mathrm{mg} / \mathrm{kg}$ of caffeine reduced times by $4.8 \%(0.8 \mathrm{~min})$ in the AA genotype compared to placebo (17.8 \pm 0.4 vs. $17.0 \pm 0.3 \mathrm{~min} ; p=0.0005)$ and $6.8 \%(1.2 \mathrm{~min})$ at $4 \mathrm{mg} / \mathrm{kg}(17.8 \pm 0.4$ vs. 16.6 $\pm 0.3 \mathrm{~min} ; p<0.0001)$. However, they did not observe any differences between 2 and 4 $\mathrm{mg} / \mathrm{kg}$ of caffeine, so the authors suggested that the consumption of 2 and $4 \mathrm{mg} / \mathrm{kg}$ improved times in $10 \mathrm{~km}$ time trial, but only in AA genotype subjects, while it had no effect on the AC genotype and reduced performance at a dose of $4 \mathrm{mg} / \mathrm{kg}$ in the CC genotype. A randomized study by Pataky et al. [107] reported that, besides the genotype shown by CYPA12, the effects of caffeine were associated with the circadian rhythm in a $3 \mathrm{~km}$ cycling test. The study revealed that performance was not only influenced by genetic factors at higher doses of $6 \mathrm{mg} / \mathrm{kg}$, along with early activity before 10 a.m., since AC heterozygotes experienced greater performance gains with caffeine ingestion than AA homozygotes, albeit without producing statistically significant results $(p=0.12)$. This shows that factors other than genetic (genotype) and circadian (time of day) parameters affect the ergogenic value of caffeine consumption and may facilitate the more personalized prescription of caffeine ingestion strategies in order to maximize performance. In a randomized study conducted by Womack et al. [105] among trained cyclists administered caffeine or placebo before completing a $40 \mathrm{~km}$ time trial, the authors observed a greater reduction in times among caffeine-doped AA homozygotes (4.9\%; placebo: $76.1 \pm 5.8 \mathrm{~min}$; caffeine: $72.4 \pm 4.2 \mathrm{~min}$ ) compared to C-allele carriers (1.8\%; placebo: $72.2 \pm 4.2 \mathrm{~min}$; caffeine: $70.9 \pm 4.3 \mathrm{~min}$ ) $(p<0.05)$. These results suggest that homozygous individuals for the A allele of this polymorphism can experience a greater ergogenic effect after ingesting caffeine.

Continuing with more cycling tests in endurance sports, a recent double-blind, crossover study performed by Carswell et al. [108] with dose at $3 \mathrm{mg} / \mathrm{kg}$ of caffeine, reported an increase in the cognitive effects for "fast" CYP1A2 metabolizers against "slow" metabolizers with respect to reaction times during exercise $(-18 \pm 9 \mathrm{vs} .-1.0 \pm 11 \mathrm{~ms})$, fastest $10 \%$ reaction time at rest $(-18 \pm 11$ vs. $-3 \pm 15 \mathrm{~ms})$ and lapses at rest $(-3.8 \pm 2.7$ vs. $+0.4 \pm 0.9)$ $(p<0.05)$, while there were no differences among the ADORA2A genotypes $(p>0.05)$.

Regarding resistance-trained athletes, they can experience acute improvements in resistance exercise, jumping and sprinting performance after consuming caffeine in conjunction with power tests, as shown in the study by Grgic et al. [28] who examined the acute effect of caffeine ( $3 \mathrm{mg} / \mathrm{kg}$ of body mass) compared to placebo. The authors found caffeine enhanced movement velocity and power output across all loads (effect size (ES): $0.20-0.61$; $p<0.05$ ); the quality and quantity of repetitions completed at $85 \%$ of one-repetition maximum (ES: $0.27-0.85 ; p<0.001$ ); vertical jump height (ES: $0.15 ; p=0.017$ ); and power output in the Wingate test (ES: $0.33-0.44 ; p<0.05)$. However, they did not find a significant 
interaction effect between CYP1A2 genotype and caffeine intake ( $p$-values ranged from 0.094 to 0.994$)$ in the performance results analyzed in this group of athletes.

When analyzing the effects of polymorphism in CYP1A2 and ADORA2A on performance, we start to observe contradictions in team sports, such as the recent study among professional handball players by Muñoz et al. [19], showing that the ergogenic response to acute caffeine intake was not modulated by CYP1A2 or ADORA2A genotypes, with just one genotype $x$ treatment interaction for ball throwing from $7 \mathrm{~m}(p=0.037)$, indicating that the ergogenic effect of caffeine in this test was greater in CYP1A2 AA homozygotes than in C-allele carriers. There were no genotype $x$ treatment interactions for either CYP1A2 or ADORA2A in the remaining variables. Collectively, caffeine increased CMJ height, performance in the sprint velocity test and ball throwing velocity from $9 \mathrm{~m}(2.8-4.3 \%$; $p=0.001-0.022$; effect size: $0.17-0.31$ ) as a whole group. Similarly, in elite basketball players, Puente et al. [30] found that polymorphism CYP1A2 c.-163C $>$ A had a minimal effect on ergogenic derived from consuming a moderate dose of caffeine $(3 \mathrm{mg} / \mathrm{kg})$ compared to placebo. Caffeine only increased Abalakov jump height by a mean of $2.9 \pm 3.6 \%$ in AA homozygotes $(p=0.03)$, while this result did not reach statistical significance for C-allele carriers $(2.3 \pm 6.8 \% ; p=0.33)$, it did not affect sprint time in the CODAT test in either genotype, but it increased the number of impacts during a simulated game in AA homozygotes $(4.1 \pm 5.3 \% ; p=0.02)$ and C-allele carriers $(3.3 \pm 3.2 \% ; p=0.01)$.

Another randomized study with adolescents ( $15 \pm 2$ years) published by Spineli et al. [109] found that a caffeine dose of $6 \mathrm{mg} / \mathrm{kg}$ increased number of sit-ups ( $35 \pm 8 \mathrm{vs} .37 \pm 9$ ), pushups $(24 \pm 11 \mathrm{vs.} 26 \pm 11)$ and distance in the Yo-Yo IR1 test $(903.2 \pm 325.7 \mathrm{vs.} 1010.4 \pm 378.9 \mathrm{~m})$ (all $p<0.05$ ), but did not influence handgrip strength ( $33.7 \pm 8.7$ vs. $35.1 \pm 8.9 \mathrm{kgf}$ ), CMJ $(47.9 \pm 13.8$ vs. $49.3 \pm 12.6 \mathrm{~cm})$, spike jump height $(52.9 \pm 14.5$ vs. $54.2 \pm 13.6 \mathrm{~cm})$ and time in agility test $(15.9 \pm 1.3$ vs. $15.8 \pm 1.1 \mathrm{~s})($ all $p>0.05)$. The authors concluded that caffeine improves muscular endurance and aerobic performance in adolescent athletes, regardless of their CYP1A2 c.-163 C>A genotype. However, Salinero et al. conducted a randomized pilot study [106] in a healthy population and reported that CYP1A2 variations did not modify the benefits or disadvantages of caffeine during exercise at a dose of $3 \mathrm{mg} / \mathrm{kg}$, as caffeine consumption increased peak power (667 $\pm 137 \mathrm{vs.} 682 \pm 140 \mathrm{~W} ; p=0.008)$ and mean power during the Wingate test $(518 \pm 111$ vs. $527 \pm 111 \mathrm{~W} ; p<0.001)$ without any differences between AA homozygotes and $\mathrm{C}$-allele carriers $(p>0.05)$. The reaction times were similar between caffeine and placebo ( $269 \pm 71$ vs. $276 \pm 31 \mathrm{~ms} ; p=0.681)$, again without any differences between AA homozygotes and C-allele carriers $(p>0.05)$. A recent randomized study by Glaister et al. [110] concluded that cyclists who took $5 \mathrm{mg} / \mathrm{kg}$ of caffeine achieved a significant time reduction in a time trial (placebo: $30.8 \pm 2.3 \mathrm{~min}$; caffeine: $29.7 \pm 1.8 \mathrm{~min} ; p<0.05$ ), but there was no effect associated with the genotype for either CYP1A2 or ADORA2A. During submaximal exercise, compared to placebo, caffeine reduced mean heart rate by $2.9 \pm 3.7 \mathrm{bpm}$ $(p<0.05)$, with effects that dissipated as exercise intensity increased, although there was no relation to genotype.

One study report on caffeine consumption derived from energy drinks in cyclists, presented by Davenport et al. [111]. They found that a $200 \mathrm{mg}$ supplement of caffeine $35 \mathrm{~min}$ before exercise seemed optimal to improve performance in a time trial, reducing the perception of effort in which the individuals with genotype AA of CYP1A2.

Future studies with larger samples are required to fully elucidate this area of research. Table 2 shows the detailed results regarding the correlation between caffeine consumption and its association with genetics. 
Table 2. Characteristics of studies referring to the association between energy drinks and genetics.

\begin{tabular}{|c|c|c|c|c|c|c|c|}
\hline \multicolumn{8}{|c|}{ Statistically Significant Positive Association } \\
\hline First Author/Country/ & Year & Study Design & Sample (n) & Gender/Age/Mean Age & Caffeine dosage & Exercise Performance test & Result \\
\hline $\begin{array}{l}\text { Womack et al./the USA } \\
{[105]}\end{array}$ & 2012 & $\begin{array}{l}\text { Randomly, double-blind, } \\
\text { placebo-controlled trial }\end{array}$ & $\begin{array}{c}35 \\
\text { (Trained cyclist) }\end{array}$ & Male, $25.0 \pm 7.3 \mathrm{y}$ & $\begin{array}{l}6 \mathrm{mg} / \mathrm{Kg} \mathrm{BM} \text { of anhydrous } \\
\text { caffeine or a placebo }\end{array}$ & $\begin{array}{l}\text { Simulated } 40-\mathrm{km} \text { time trials } \\
\text { on a cycle ergometer }\end{array}$ & $\begin{array}{c}\text { Caffeine supplementation reduced } \\
\text { 40-kilometre time }(p<0.05) \text { in AA } \\
\text { homozygotes }(4.9 \% ; \\
\text { caffeine }=72.4 \pm 4.2 \mathrm{~min}, \\
\text { placebo }=76.1 \pm 5.8 \mathrm{~min}) \text { compared } \\
\text { to } C \text { allele carriers }(1.8 \% ; \\
\text { caffeine }=70.9 \pm 4.3 \mathrm{~min}, \\
\text { placebo }=72.2 \pm 4.2 \mathrm{~min})\end{array}$ \\
\hline Guest et al./Canada [29] & 2018 & $\begin{array}{l}\text { Split-plot randomized, } \\
\text { double-blinded, } \\
\text { placebo-controlled }\end{array}$ & $\begin{array}{c}101 \\
\text { (Competitive athletes) }\end{array}$ & Male, $25 \pm 4$ y & $0-2-4 \mathrm{mg} / \mathrm{Kg}$ caffeine & 10-km cycling time trial & $\begin{array}{c}\text { AA genotype decreased time } 4.8 \% \text { at } \\
2 \mathrm{mg} / \mathrm{Kg}(17.0 \pm 0.3 \mathrm{vs} \\
17.8 \pm 0.4 \mathrm{~min}, p=0.0005) \text { and } 6.8 \% \\
\text { at } 4 \mathrm{mg} / \mathrm{Kg}(16.6 \pm 0.3 \mathrm{vs} . \\
17.8 \pm 0.4 \mathrm{~min}, p<0.0001) . \mathrm{CC} \\
\text { genotype, } 4 \mathrm{mg} / \mathrm{Kg} \text { increased } \\
\text { cycling time } 13.7 \%(20.8 \pm 0.8 \mathrm{vs} \text {. } \\
18.3 \pm 0.5 \mathrm{~min}, p=0.04) \text {. Among } \\
\text { AA } / \mathrm{CC} \text { with the AC genotype time } \\
\text { decreased } 4.8 \% \text { at } 2 \mathrm{mg} / \mathrm{Kg} \\
(17.0 \pm 0.3 \mathrm{vs} .17 .8 \pm 0.4 \mathrm{~min} \\
p=0.0005) \text { and } 6.8 \% \text { at } 4 \mathrm{mg} / \mathrm{Kg} \\
(16.6 \pm 0.3 \mathrm{vs} .17 .8 \pm 0.4 \mathrm{~min}, \\
p<0.0001) . \\
\text { Significant }(p<0.0001) \text { caffeine-gene } \\
\text { interaction was observed. } 4 \mathrm{mg} / \mathrm{Kg} \\
\text { caffeine decreased cycling time by } \\
3 \% \text { versus placebo }(17.6 \pm 0.1 \mathrm{vs} . \\
18.1 \pm 0.1 \mathrm{~min}, p=0.01)\end{array}$ \\
\hline Puente et al./Spain [30] & 2018 & $\begin{array}{l}\text { Case-control ecological } \\
\text { experimental }\end{array}$ & $\begin{array}{c}19 \\
\text { (Elite basketball players) }\end{array}$ & Both, $26.7 \pm 3.5 \mathrm{y}$ & $3 \mathrm{mg} / \mathrm{Kg}$ of caffeine & $\begin{array}{l}\text { Abalakov jump test followed } \\
\text { by the CODAT test. } 20 \text {-min } \\
\text { simulated basketball game }\end{array}$ & $\begin{array}{c}\text { Caffeine intake increased Abalakov } \\
\text { jump height by a mean of } 2.9 \pm 3.6 \% \\
\text { in AA homozygotes }(p=0.03) \text { while } \\
\text { this effect did not reach statistical } \\
\text { significance for C-allele carriers } \\
\text { (2.3 } \pm 6.8 \%, p=0.33) \text {. } \\
\text { The number of impacts during the } \\
\text { simulated game also increased in } \\
\text { both AA homozygotes }(4.1 \pm 5.3 \% \text {, } \\
p=0.02) \text { and C-allele carriers } \\
(3.3 \pm 3.2 \%, p=0.01) .\end{array}$ \\
\hline $\begin{array}{c}\text { Carswell et al./the UK } \\
\text { [108] }\end{array}$ & 2020 & $\begin{array}{l}\text { Double-blind, } \\
\text { placebo-controlled } \\
\text { crossover }\end{array}$ & $\begin{array}{c}18 \\
\text { (Health adults) }\end{array}$ & Both, $24 \pm 4$ y & $3 \mathrm{mg} / \mathrm{Kg}$ of caffeine & $\begin{array}{l}\text { 15-min cycling time trial and } \\
\text { cognitive performance PVT } \\
\text { pre, } 50 \text { and } 95-\text {-min } \\
\text { post-supplementation) }\end{array}$ & $\begin{array}{c}\text { Caffeine enhanced exercise } \\
\text { performance }(p<0.001), \text { but effects } \\
\text { were not different between } \\
\text { participants with ADORA2A 'high } \\
\text { metabolizers' vs. 'low' sensitivity } \\
\text { genotype }(+6.4 \pm 5.8 \% \text { vs. } \\
+8.2 \pm 6.8 \%) \text {, or } C Y P 1 A 2 \text { 'fast } \\
\text { metabolizers' vS. 'slow' metabolism } \\
\text { genotype }(+7.2 \pm 5.9 \mathrm{vss}+7.0 \pm 6.7 \% \text {, } \\
p>0.05)\end{array}$ \\
\hline
\end{tabular}


Table 2. Cont

\begin{tabular}{|c|c|c|c|c|c|c|c|}
\hline \multicolumn{8}{|c|}{ Statistically Significant Positive Association } \\
\hline First Author/Country/ & Year & Study Design & Sample (n) & Gender/Age/Mean Age & Caffeine dosage & Exercise Performance test & Result \\
\hline Grgic et al./Australia [28] & 2020 & $\begin{array}{l}\text { Double-blind, randomized, } \\
\text { crossover }\end{array}$ & $\begin{array}{c}22 \\
\text { (Resistance-trained } \\
\text { participants) }\end{array}$ & $\begin{array}{l}\text { Male, } 27.0 \pm 5.6 \text { AA group; } \\
29.8 \pm 3.6 \text { CT /TT group }\end{array}$ & $3 \mathrm{mg} / \mathrm{Kg}$ of caffeine & $\begin{array}{l}\text { Movement velocity, power } \\
\text { output in the bench press, } \\
\text { quality, and quantity of } \\
\text { performed repetitions in the } \\
\text { bench press exercise, vertical } \\
\text { jump height in a CMJ test } \\
\text { and power output in a } \\
\text { Wingate test }\end{array}$ & $\begin{array}{c}\text { Caffeine ingestion enhanced } \\
\text { movement velocity and power } \\
\text { output across all loads (ES: } 0.20-0.61 \text {, } \\
p<0.05 \text {, the quality and quantity of } \\
\text { performed repetitions with } 85 \% \text { of } \\
1 \text { RM (ES: } 0.27-0.85, p<0.001 \text { for all), } \\
\text { vertical jump height (ES: } 0.15, \\
p=0.017 \text { ) and power output in the } \\
\text { Wingate test (ES: } 0.33-0.44, p<0.05 \\
\text { for all genotypes) }\end{array}$ \\
\hline \multicolumn{8}{|c|}{ No statistically significant association } \\
\hline Pataky et al./the USA [107] & 2016 & $\begin{array}{c}\text { Randomly } \\
\text { counterbalanced, } \\
\text { double-blind, } \\
\text { placebo-controlled }\end{array}$ & $\begin{array}{c}38 \\
\text { (Recreational trained } \\
\text { cyclists) }\end{array}$ & Male, $21 \pm 1$ y & $\begin{array}{c}6 \mathrm{mg} / \mathrm{Kg} \text { of caffeine } \\
\text { Additionally, } 25 \mathrm{~mL} \text { of } \\
1.14 \% \text { caffeine or placebo } \\
\text { solution were mouth } \\
\text { rinsed before each time } \\
\text { trial }\end{array}$ & 3-km simulated time trials & $\begin{array}{c}\text { No association in endurance } \\
\text { performance in CYY1A A genotypes } \\
\text { vs placebo, but favoring AC } \\
\text { genotype }(5.1 \% \pm 6.1 \%, p=0.12) \text { vs } \\
\text { placebo }\end{array}$ \\
\hline Salinero et al./Spain [106] & 2017 & $\begin{array}{c}\text { Double-blind randomized } \\
\text { experimental }\end{array}$ & $\begin{array}{c}21 \\
\text { (Healthy participants) }\end{array}$ & Both, $29.3 \pm 7.7 \mathrm{y}$ & $3 \mathrm{mg} / \mathrm{Kg}$ of caffeine & $\begin{array}{l}30 \mathrm{~s} \text { Wingate test, visual } \\
\text { attention, and side effects }\end{array}$ & $\begin{array}{c}\text { No differences in reaction times } \\
\text { between caffeine and placebo } \\
\text { conditions (276 } \pm 31 \text { milliseconds vs. } \\
269 \pm 71 \text { milliseconds, } p=0.681) \\
\text { between AA homozygotes and } \\
\text { C-allele carriers. } \\
31.3 \% \text { of the C-allele carriers } \\
\text { reported increased nervousness after } \\
\text { caffeine ingestion, while none of the } \\
\text { AA homozygotes reported them. } \\
\text { Caffeine ingestion increased peak } \\
\text { power }(682 \pm 140 \mathrm{~W} \text { vs. } \\
667 \pm 137 \mathrm{~W}, p=0.008) \text { and mean } \\
\text { power during the Wingate test } \\
(527 \pm 111 \mathrm{~W} \text { vs. } 518 \pm 111 \mathrm{~W}, \\
p<0.001)\end{array}$ \\
\hline $\begin{array}{c}\text { Davenport et al./the UK } \\
\text { [111] }\end{array}$ & 2020 & $\begin{array}{l}\text { Double-blind, } \\
\text { four-treatment, randomly, } \\
\text { crossover }\end{array}$ & $\begin{array}{c}13 \\
\text { (Well-trained cyclists) }\end{array}$ & Both, $28 \pm 2 y$ & $\begin{array}{c}200 \mathrm{mg} \text { of caffeine, } 1600 \\
\text { mg of } \beta \text {-alanine and } 1000 \\
\text { mg quercetin }\end{array}$ & $\begin{array}{l}30 \text { min of steady-state } \\
\text { exercise on a cycle ergometer } \\
\text { followed by a 15-min } \\
\text { time trial }\end{array}$ & $\begin{array}{l}\text { Caffeine supplementation appeared } \\
\text { optimal for improved performance } \\
\text { in a subsequent fatiguing time trial } \\
\text { without statistical differences } \\
(p>0.05) \\
\end{array}$ \\
\hline
\end{tabular}


Table 2. Cont

\begin{tabular}{|c|c|c|c|c|c|c|c|}
\hline \multicolumn{8}{|c|}{ Statistically Significant Positive Association } \\
\hline First Author/Country/ & Year & Study Design & Sample (n) & Gender/Age/Mean Age & Caffeine dosage & Exercise Performance test & Result \\
\hline Muñoz et al./Spain [19] & 2020 & $\begin{array}{l}\text { Double-blind, } \\
\text { placebo-controlled, } \\
\text { crossover }\end{array}$ & $\begin{array}{c}31 \\
\text { (Professional handball } \\
\text { players) }\end{array}$ & Both, $23.7 \pm 2.8 \mathrm{y}$ & $3 \mathrm{mg} / \mathrm{Kg}$ of caffeine & $\begin{array}{l}\text { CMJ test, a sprint test, an } \\
\text { agility test, an isometric } \\
\text { handgrip test, and several } \\
\text { ball throws }\end{array}$ & $\begin{array}{l}\text { There were no genotype } \mathrm{x} \text { treatment } \\
\text { interactions for CYP1A2 or for } \\
\text { ADORA2A }(p<0.05) \text {, only for the } \\
\text { ball throwing from } 7 \mathrm{~m}(p=0.037), \\
\text { higher in CYP1A2 AA homozygotes } \\
\text { than in C-allele carriers }\end{array}$ \\
\hline Glaister et al./the UK [110] & 2020 & $\begin{array}{l}\text { Randomized, double-blind, } \\
\text { placebo controlled }\end{array}$ & $\begin{array}{c}66 \\
\text { (Trained cyclist) }\end{array}$ & Male, $41.9 \pm 8.6$ y & $\begin{array}{l}5 \mathrm{mg} / \mathrm{Kg} \text { of } \mathrm{BM} \text { of caffeine } \\
\text { or placebo one hour before } \\
\text { performance test }\end{array}$ & $\begin{array}{l}\text { Incremental cycling test, } \\
\text { followed by } \pm 30 \text { min of } \\
\text { time-trial }\end{array}$ & $\begin{array}{l}\text { Caffeine reduced the time to } \\
\text { complete the time-trial, without } \\
\text { effect of genotype (caffeine: } \\
29.7 \pm 1.8 \mathrm{~min} ; \text { placebo: } \\
30.8 \pm 2.3 \mathrm{~min}(p<0.05)) \text {; During } \\
\text { submaximal exercise, caffeine } \\
\text { reduced mean heart rate by } \\
2.9 \pm 3.7 \mathrm{~b} / \mathrm{min}, \text { and also reduced } \\
\text { perceived exertion by } 0.5 \pm 0.8, \text { and } \\
\text { increased blood lactate by } \\
0.29 \pm 0.42 \mathrm{mmol} / \mathrm{L}, \text { respiratory } \\
\text { exchange ratio by } 0.013 \pm 0.032, \text { and } \\
\text { minute ventilation by } \\
3.1 \pm 6.8 \mathrm{~L} / \mathrm{min} \text {. }\end{array}$ \\
\hline
\end{tabular}

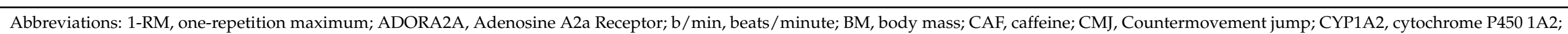

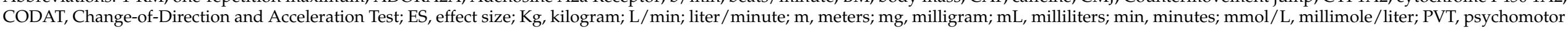
vigilance test; PLA, placebo; $\mathrm{VO}_{2} \max$, maximal oxygen consumption; $\mathrm{W}$, Watts; $\mathrm{y}$, years. 


\section{Discussion}

The results of the studies analyzed herein are ambiguous or even contradictory. This discrepancy is mainly due to the influence of genetics on the ergogenic effects of caffeine, particularly when the correlation between caffeine and athletic performance appears to be more evident and validated in the scientific literature. Due to the lack of studies, there are still some confounding factors which may contribute to these discrepancies in the results.

\subsection{Energy Drinks and Relationship in Sports Performance}

Caffeine is the main component of energy drinks, as mentioned previously. As such, to understand the effects of energy drinks we need to understand the mechanisms that this phytochemical produces in the human body. Caffeine is absorbed rapidly, reaching its peak plasma concentration in 30-120 min [112]. Its absorption rate is regulated by various factors such as the pharmacokinetics in the function of cytochrome activity, mainly those which metabolize caffeine in the liver and above all enzyme CYP1A2. In pharmacological terms, it has been shown that consuming more than $6 \mathrm{mg}$ of caffeine per $\mathrm{kg}$ of body mass seems to saturate hepatic caffeine metabolism, as stronger effects are not observed when greater amounts are ingested [113].

The consumption of caffeine stimulates the central nervous system through the blockade of peripheral and cerebral adenosine receptors, thus generating a delay in the onset of fatigue [114]. Caffeine also stimulates motor neurons by increasing their recruitment [115]. Its effect is also associated with an increased release of $\mathrm{Ca}^{2+}$ stored in intracellular neuronal reserves, triggering a range of important neuronal processes [116]. Caffeine enhances the mobilization of glycerol and free acids in blood [117], thereby increasing fat oxidation during exercise at low and moderate loads [118]. Furthermore, caffeine stimulates adrenaline (epinephrine) secretion. This response, together with all those mentioned above, produces a series of metabolic changes associated with ergogenic effects that illustrate its consumption in athletic performance [16].

With respect to caffeine's different mechanisms of action once ingested by humans, its use is considered an ergogenic aid to increase sporting performance [119] and improve cognitive aspects such as concentration, alertness and reaction time [120]. In addition, energy drinks possess effective doses that trigger these stimulating consequences in the human body, so it is not surprising that the consumption of such drinks and their inclusion of taurine and other phytochemicals exhibits a positive correlation with physical performance in many different sporting movements, disciplines and sports (Table 1).

On the other hand, we also found several studies that did not observe a positive association between the consumption of energy drinks combining caffeine and taurine and improved athletic performance, even with the risk they may pose to the nervous system of those who consume them [121]. The reasons why these studies did not evidence any positive associations with increased physical performance could be because their subjects were already habituated to caffeine consumption [78,122], because of the subjects' high level of training, or due to a lack of statistical power arising from small sample sizes.

A systematic review and meta-analysis [9] were published in 2017 to study the ergogenic effects of energy drinks and different expressions of sporting performance. The authors concluded that the analyzed literature demonstrated a significant improvement in athletic performance in tests of muscle strength, jumps, resistance exercises and specific actions performed in distinct sporting disciplines and that drinks which contained taurine significantly increased these effects. They also highlighted the need for more research into the area and more control in the testing protocols. This review confirms the data published in the literature and completes the findings reported to date.

\subsection{Energy Drinks and Relationship in Cardiovascular Risk}

Adolescents, young adults and above all athletes are drawn towards energy drinks because of their perceived benefits. However, these individuals are often unaware of the harmful effects associated with these drinks. The body gets used to consuming these drinks 
in order to function and maintain a level of performance [60,84]. Although a plethora of studies have shown that energy drinks are more effective at enhancing cognitive function or increasing energy levels compared to traditional soft drinks, their excessive consumption can be detrimental to both an individual's athletic performance and their health [85]. The use of energy drinks, coffee and other caffeinated drinks as a substitute for sleep in relation to school, sporting or day-to-day duties has developed into a regular habit among adolescents and young adults, giving rise to particular concern about the risk such consumption could imply for the population's health [84]. Numerous studies have evidenced the risks of cardiovascular diseases that derive from constant dependence in an otherwise healthy population [86,87], including hypertension, tachycardia and even sudden death from lethal doses of $5 \mathrm{~g}$, equivalent to approximately 42 cups of coffee with $120 \mathrm{mg}$ caffeine/cup [90], or just $3 \mathrm{~g}$ [92].

The ergogenic dose of caffeine necessary to improve neuromuscular performance during sport depends on the magnitude and duration of the activity. Researchers have shown that $3 \mathrm{mg} / \mathrm{kg}$ is enough to improve muscle actions in strength and endurance sports, yet a higher dose $(9 \mathrm{mg} / \mathrm{kg})$ is associated with the appearance of unwanted side effects [123]. At the same time, a healthy adult can consume up to $400 \mathrm{mg}$ of caffeine/day (equivalent to $6 \mathrm{mg} / \mathrm{kg}$ in individuals weighing less than $65 \mathrm{~kg}$ ) without producing any adverse effects [93]. Studies which have reported changes in heart rate and blood pressure involved the supplementation of caffeine with ephedrine in synergy, such as the works of Haller et al. [97] and Shekelle et al. [95], which observed increases in heart rate and blood pressure and weight loss, respectively, but at moderate doses (200 mg of caffeine and $25 \mathrm{mg}$ of ephedrine) and without the participants reporting any adverse events. Similar results were found in the study of Nowak et al. [124], in which, after ingesting energy drinks containing $80 \mathrm{mg}$ of caffeine, glucose, taurine, vitamins and other ingredients glucuronide, acute intake of energy drinks can increase diastolic blood pressure by more than $8 \%$, blood sugar and discomfort level of healthy young people. Besides, Hajsadeghi et al. [125] confirmed that drinking energy drinks (caffeinated energy drinks) before and at a specific time point of $4 \mathrm{~h}$ may lead to decreased heart rate and more frequent ST-segment and T-wave (ST-T) changes in healthy young people.

In addition, Red Bull ${ }^{\circledR}$ has the same effect on blood pressure as a considerable amount of caffeine, this increase occurs through different hemodynamic pathways. Reed Bull mainly affects cardiac parameters, while caffeine mainly causes vascular effects. In addition, the auxiliary components of Red Bull ${ }^{\circledR}$ (taurine, glucuronide, and group B vitamins) do not seem to affect these pathways [126].

To date, no studies have recorded any severe adverse events in athletes related to energy drink use, with the doses that are currently used by this population ideal for enhancing sporting performance without provoking harm thanks to the control exerted by the WADA [34].

\subsection{Energy Drinks, Genetics, and the Relationship with Athletic Performance; Future Prospects}

The influence of the genes CYP1A2 and ADORA2A on the body's response to caffeine has been discussed in detail and there is a general overview in the current literature. The role of these two genes can explain a significant proportion of the interindividual variation in performance following caffeine ingestion reported in studies [26]. By determining the extent to which these genes and any new polymorphisms discovered in the future can moderate an individual's response to caffeine during exercise, we will be able to guarantee that caffeine supplementation programs can be tailored to each athlete in order to maximize the potential ergogenic effect of energy drinks [27].

Several randomized, placebo-controlled studies have published data that feature subjects' inhomogeneous groups in terms of sporting level, age and sex for both the caffeine and placebo cohorts $[19,28-30,105-110]$. Most studies reviewed in the present work that examined the link between genetics and athletic performance in strength and endurance events observed that polymorphism c.-163C >A (rs762551) in gene CYP1A2 presents an 
association with improved performance, while they did not observe any ergogenic effects in relation to gene ADORA2A. It has been shown that the most effective dose to improve sports performance in association with genetics is around $3 \mathrm{mg} / \mathrm{kg}$ of body weight (equivalent to $200 \mathrm{mg}$ of caffeine per energy drink), as this enhances endurance performance in cycling [29], reduces reaction times and improves cognitive performance [108], and augments power [28], as observed in team sports, such as handball [19] and basketball [30], where improvements in various tests were reported in association with polymorphism of CYP1A2. In turn, a dose of 5-6 mg/ $\mathrm{kg}$ of body weight (equivalent to $400-500 \mathrm{mg}$ of caffeine), particularly in cycling $[105,107,110]$, is known to prolong the ergogenic effects in certain aspects of endurance events (cycling, athletics, triathlon) and therefore sustain improved performance. Furthermore, genetic factors are known to maintain this performance in competitions, data which should be studied in these events in the future, as explained by Grgic et al. [31]. In their review of the ergogenic effects of caffeine associated with polymorphism in CYP1A2, the authors found few studies that reported a better response to caffeine in terms of sporting performance among the AA genotype subjects, observing that variations in this gene can modular the ergogenic effects of caffeine, but differences between genotypes were small, inconsistent or limited to specific exercises. Grgic's findings agree with those presented in our review, highlighting areas for future research in order to amplify the information on genetics and improved athletic performance with the use of energy drinks.

Polymorphism c.1976T>C (rs5751876) in gene ADORA2A has been shown to modulate sleep-wake activity $[99,100]$. Yet when studied in conjunction with a polymorphism in CYP1A2 the results did not reveal a clear relationship with performance, concentration or states of nervousness, as indicated by Carswell et al. [108]. While self-reported insomnia, diuresis and excessive activity was documented in handball players with TT genotype [19] and an ergogenic response to caffeine consumption has recently been observed in C-allele carriers [127].

Successive studies with larger sample sizes should be conducted to address the discrepancies between genetic condition and sports performance that have been exposed in the scientific literature.

According to the recent review of caffeine in the context of athletic performance published by Martins et al. [128] improvements in performance were noted at doses of $2-9 \mathrm{mg} / \mathrm{kg}$. This is notable because the physiological mechanisms that are involved when the dose is increased remain unclear, since the subject's regular consumption and the time of day when the caffeine is taken may have positive or negative effects on the ensuing benefits. Recent findings show that caffeine can enhance or diminish performance during exercise. These antagonistic responses may even occur when using the same dose and in individuals with the same characteristics. These effects are subdivided into those linked to the caffeine itself, daily consumption habits, physiological factors and genetic factors. A range of hypotheses has been put forward that indicate the genetic influences related to polymorphisms in the genes CYP1A2 and ADORA2A. Current evidence suggests that CYP1A2 only has a strong influence in endurance activities, where the TT genotype has greater benefits upon increasing the caffeine dose, while individuals of CC genotype suffer a decline in performance with the use of caffeine. On the other hand, there do not appear to be any differences between responses with respect to the polymorphism in CPY1A2 in intermittent and anaerobic exercises, but only a few studies with limited participants have been completed, which underlines this is an interesting area for future studies. Another potential focal point where there is a lack of results is the caffeine ingestion time before carrying out short tests [129], with the ergogenic effect of caffeine known to develop 1-3 h after consumption, as highlighted by the present review and in accordance with the 2002 study by Bell et al. [130]. It is important to note that there are only ambiguous results in the current literature concerning physical characteristics (training, sex, etc.), caffeine abstinence and the possible impact of polymorphism in gene ADORA2A. All the factors in this review should be taken into account when designing studies that aim to improve the current body 
of research, as described in this section on future prospects for an improved understanding of the effects of caffeine, its ergogenic effects and the role of genetics.

Year after year, new polymorphisms are discovered to have a bearing on different aspects of athletic performance, with as many as 120 polymorphisms known to hold a direct relationship with the performance [131]. According to a recent review by Joyner [132], we are still a long way from developing a complete understanding of genetics' influence on sporting performance, and in the future, we might be able to model it using field tests, which, with more variables, could be highly predictive of an athlete's performance. In this context, a recent study of interest is that of Varillas et al. [133], which looked at another cytochrome P450 gene (CYP2D6) and variants in the glutathione S-transferase family (GSTM, GSTP, and GSTP), as it found "favorable" frequencies in endurance athletes compared to a control population using genetic scoring, as Joyner explains, to determine which are the most useful in terms of predicting performance. Such information on these hepatic variables would be of interest to promote genetic scoring in various genes involved in hepatic metabolism and which affect caffeine's action-such as CYP1A2, ADORA2A, CYP2D6, and the glutathione S-transferase (GST) family-to expand on current knowledge and help resolve some of the contrasting results in the literature. Genetic scoring could represent a more powerful tool and means of discovering the weight of each polymorphism on the metabolism of caffeine absorbed from energy drinks and its ergogenic effects in endurance sports and strength tests [31].

\section{Conclusions}

Energy drinks mainly comprised of caffeine and taurine demonstrate a positive effect in terms of enhancing sporting performance, although several studies did not report any such effect. The exact mechanism of caffeine's ergogenic effect during exercise is still relatively unknown; the same is true for the risks its consumption may pose to the health of athletes, as it can provoke multifactorial adverse events. The field has received insufficient attention and requires further investigation to answer all of these questions. Nevertheless, the increase in athletic performance may also be related to alterations in perceived effort, reaction time, cognition and/or mood. There is a dearth of studies that confirm caffeine's effectiveness in sports such as sprinting, athletics, football, tennis, handball, and hockey, plus a lack of research on the role of genetics, where the results appear to contradict each other. Therefore, we need to study more cytochromes involved in caffeine metabolism to open new lines in our understanding of the association between genetics and athletic performance following the consumption of caffeine and, in turn, energy drinks.

Author Contributions: Conceptualization, J.G.-H. and D.V.-D.; writing-original draft preparation, J.G-H. and D.V.-D., writing - review and editing, J.G.-H. and D.V.-D. All authors have read and agreed to the published version of the manuscript.

Funding: This research received no external funding.

Institutional Review Board Statement: Not applicable.

Informed Consent Statement: Not applicable.

Data Availability Statement: No new data were created or analyzed in this study. Data sharing is not applicable to this article.

Conflicts of Interest: The authors declare no conflict of interest.

\section{References}

1. Ali, F.; Rehman, H.; Babayan, Z.; Stapleton, D.; Joshi, D.D. Energy drinks and their adverse health effects: A systematic review of the current evidence. Postgrad. Med. 2015, 127, 308-322. [CrossRef] [PubMed]

2. Kumar, G.; Park, S.; Onufrak, S. Perceptions about energy drinks are associated with energy drink intake among U.S. youth. Am. J. Health Promot. 2015, 29, 238-244. [CrossRef] [PubMed]

3. Higgins, J.P.; Babu, K.; Deuster, P.A.; Shearer, J. Energy Drinks: A Contemporary Issues Paper. Curr. Sports Med. Rep. 2018, 17, 65-72. [CrossRef] [PubMed] 
4. Porrini, M.; Del Bo', C. Ergogenic Aids and Supplements. Front. Horm. Res. 2016, 47, 128-152. [CrossRef] [PubMed]

5. Kerksick, C.M.; Wilborn, C.D.; Roberts, M.D.; Smith-Ryan, A.; Kleiner, S.M.; Jäger, R.; Collins, R.; Cooke, M.; Davis, J.N.; Galvan, E.; et al. ISSN exercise \& sports nutrition review update: Research \& recommendations. J. Int. Soc. Sports Nutr. 2018, 15, 38. [CrossRef]

6. Bishop, D. Dietary supplements and team-sport performance. Sports Med. 2010, 40, 995-1017. [CrossRef]

7. McDowall, J.A. Supplement use by Young Athletes. J. Sports Sci. Med. 2007, 6, 337-342.

8. Rawson, E.S.; Miles, M.P.; Larson-Meyer, D.E. Dietary Supplements for Health, Adaptation, and Recovery in Athletes. Int. J. Sport Nutr. Exerc. Metab. 2018, 28, 188-199. [CrossRef]

9. Souza, D.B.; Del Coso, J.; Casonatto, J.; Polito, M.D. Acute effects of caffeine-containing energy drinks on physical performance: A systematic review and meta-analysis. Eur. J. Nutr. 2017, 56, 13-27. [CrossRef] [PubMed]

10. Astorino, T.A.; Roberson, D.W. Efficacy of acute caffeine ingestion for short-term high-intensity exercise performance: A systematic review. J. Strength Cond. Res. 2010, 24, 257-265. [CrossRef]

11. McLellan, T.M.; Lieberman, H.R. Do energy drinks contain active components other than caffeine? Nutr. Rev. 2012, 70, 730-744. [CrossRef]

12. Gwacham, N.; Wagner, D.R. Acute effects of a caffeine-taurine energy drink on repeated sprint performance of American college football players. Int. J. Sport Nutr. Exerc. Metab. 2012, 22, 109-116. [CrossRef]

13. Lara, B.; Gonzalez-Millán, C.; Salinero, J.J.; Abian-Vicen, J.; Areces, F.; Barbero-Alvarez, J.C.; Muñoz, V.; Portillo, L.J.; GonzalezRave, J.M.; Del Coso, J. Caffeine-containing energy drink improves physical performance in female soccer players. Amino Acids 2014, 46, 1385-1392. [CrossRef] [PubMed]

14. Del Coso, J.; Muñoz-Fernández, V.E.; Muñoz, G.; Fernández-Elías, V.E.; Ortega, J.F.; Hamouti, N.; Barbero, J.C.; Muñoz-Guerra, J. Effects of a caffeine-containing energy drink on simulated soccer performance. PLoS ONE 2012, 7, e31380. [CrossRef] [PubMed]

15. Astorino, T.A.; Matera, A.J.; Basinger, J.; Evans, M.; Schurman, T.; Marquez, R. Effects of red bull energy drink on repeated sprint performance in women athletes. Amino Acids 2012, 42, 1803-1808. [CrossRef] [PubMed]

16. Graham, T.E. Caffeine and exercise: Metabolism, endurance and performance. Sports Med. 2001, 31, 785-807. [CrossRef] [PubMed]

17. Prins, P.J.; Goss, F.L.; Nagle, E.F.; Beals, K.; Robertson, R.J.; Lovalekar, M.T.; Welton, G.L. Energy Drinks Improve Five-Kilometer Running Performance in Recreational Endurance Runners. J. Strength Cond. Res. 2016, 30, 2979-2990. [CrossRef] [PubMed]

18. Del Coso, J.; Pérez-López, A.; Abian-Vicen, J.; Salinero, J.J.; Lara, B.; Valadés, D. Enhancing physical performance in male volleyball players with a caffeine-containing energy drink. Int. J. Sports Physiol. Perform. 2014, 9, 1013-1018. [CrossRef]

19. Muñoz, A.; López-Samanes, Á.; Aguilar-Navarro, M.; Varillas-Delgado, D.; Rivilla-García, J.; Moreno-Pérez, V.; Del Coso, J. Effects of CYP1A2 and ADORA2A Genotypes on the Ergogenic Response to Caffeine in Professional Handball Players. Genes 2020, 11, 933. [CrossRef]

20. Grasser, E.K.; Miles-Chan, J.L.; Charrière, N.; Loonam, C.R.; Dulloo, A.G.; Montani, J.P. Energy Drinks and Their Impact on the Cardiovascular System: Potential Mechanisms. Adv. Nutr. 2016, 7, 950-960. [CrossRef]

21. Basrai, M.; Schweinlin, A.; Menzel, J.; Mielke, H.; Weikert, C.; Dusemund, B.; Putze, K.; Watzl, B.; Lampen, A.; Bischoff, S.C. Energy Drinks Induce Acute Cardiovascular and Metabolic Changes Pointing to Potential Risks for Young Adults: A Randomized Controlled Trial. J. Nutr. 2019, 149, 441-450. [CrossRef] [PubMed]

22. Wolk, B.J.; Ganetsky, M.; Babu, K.M. Toxicity of energy drinks. Curr. Opin. Pediatr. 2012, 24, 243-251. [CrossRef] [PubMed]

23. Chrysant, S.G.; Chrysant, G.S. Cardiovascular complications from consumption of high energy drinks: Recent evidence. J. Hum. Hypertens. 2015, 29, 71-76. [CrossRef] [PubMed]

24. Pickering, C.; Grgic, J. Caffeine and Exercise: What Next? Sports Med. 2019, 49, 1007-1030. [CrossRef]

25. Guest, N.S.; Horne, J.; Vanderhout, S.M.; El-Sohemy, A. Sport Nutrigenomics: Personalized Nutrition for Athletic Performance. Front. Nutr. 2019, 6, 8. [CrossRef] [PubMed]

26. Southward, K.; Rutherfurd-Markwick, K.; Badenhorst, C.; Ali, A. The Role of Genetics in Moderating the Inter-Individual Differences in the Ergogenicity of Caffeine. Nutrients 2018, 10, 1352. [CrossRef]

27. Pickering, C.; Kiely, J. Are the Current Guidelines on Caffeine Use in Sport Optimal for Everyone? Inter-individual Variation in Caffeine Ergogenicity, and a Move Towards Personalised Sports Nutrition. Sports Med. 2018, 48, 7-16. [CrossRef]

28. Grgic, J.; Pickering, C.; Bishop, D.J.; Schoenfeld, B.J.; Mikulic, P.; Pedisic, Z. CYP1A2 genotype and acute effects of caffeine on resistance exercise, jumping, and sprinting performance. J. Int. Soc. Sports Nutr. 2020, 17, 21. [CrossRef]

29. Guest, N.; Corey, P.; Vescovi, J.; El-Sohemy, A. Caffeine, CYP1A2 Genotype, and Endurance Performance in Athletes. Med. Sci. Sports Exerc. 2018, 50, 1570-1578. [CrossRef] [PubMed]

30. Puente, C.; Abián-Vicén, J.; Del Coso, J.; Lara, B.; Salinero, J.J. The CYP1A2 -163C>A polymorphism does not alter the effects of caffeine on basketball performance. PLoS ONE 2018, 13, e0195943. [CrossRef]

31. Grgic, J.; Pickering, C.; Del Coso, J.; Schoenfeld, B.J.; Mikulic, P. CYP1A2 genotype and acute ergogenic effects of caffeine intake on exercise performance: A systematic review. Eur. J. Nutr. 2020. [CrossRef]

32. Salinero, J.J.; Lara, B.; Abian-Vicen, J.; Gonzalez-Millán, C.; Areces, F.; Gallo-Salazar, C.; Ruiz-Vicente, D.; Del Coso, J. The use of energy drinks in sport: Perceived ergogenicity and side effects in male and female athletes. Br. J. Nutr. 2014, 112, 1494-1502. [CrossRef]

33. Burke, L.M. Caffeine and sports performance. Appl. Physiol. Nutr. Metab. 2008, 33, 1319-1334. [CrossRef] [PubMed] 
34. Mottram, D.; Chester, N.; Atkinson, G.; Goode, D. Athletes' knowledge and views on OTC medication. Int. J. Sports Med. 2008, 29, 851-855. [CrossRef]

35. Wickham, K.A.; Spriet, L.L. Administration of Caffeine in Alternate Forms. Sports Med. 2018, 48, 79-91. [CrossRef] [PubMed]

36. Kamimori, G.H.; Karyekar, C.S.; Otterstetter, R.; Cox, D.S.; Balkin, T.J.; Belenky, G.L.; Eddington, N.D. The rate of absorption and relative bioavailability of caffeine administered in chewing gum versus capsules to normal healthy volunteers. Int. J. Pharm. 2002, 234, 159-167. [CrossRef]

37. Campbell, B.; Wilborn, C.; La Bounty, P.; Taylor, L.; Nelson, M.T.; Greenwood, M.; Ziegenfuss, T.N.; Lopez, H.L.; Hoffman, J.R.; Stout, J.R.; et al. International Society of Sports Nutrition position stand: Energy drinks. J. Int. Soc. Sports Nutr. $2013,10,1$. [CrossRef]

38. Pasman, W.J.; van Baak, M.A.; Jeukendrup, A.E.; de Haan, A. The effect of different dosages of caffeine on endurance performance time. Int. J. Sports Med. 1995, 16, 225-230. [CrossRef]

39. Polito, M.D.; Grandolfi, K.; de Souza, D.B. Caffeine and resistance exercise: The effects of two caffeine doses and the influence of individual perception of caffeine. Eur. J. Sport Sci. 2019, 19, 1342-1348. [CrossRef]

40. Jenkins, N.T.; Trilk, J.L.; Singhal, A.; O'Connor, P.J.; Cureton, K.J. Ergogenic effects of low doses of caffeine on cycling performance. Int. J. Sport Nutr. Exerc. Metab. 2008, 18, 328-342. [CrossRef]

41. Southward, K.; Rutherfurd-Markwick, K.J.; Ali, A. The Effect of Acute Caffeine Ingestion on Endurance Performance: A Systematic Review and Meta-Analysis. Sports Med. 2018, 48, 1913-1928. [CrossRef] [PubMed]

42. Bruce, C.R.; Anderson, M.E.; Fraser, S.F.; Stepto, N.K.; Klein, R.; Hopkins, W.G.; Hawley, J.A. Enhancement of 2000-m rowing performance after caffeine ingestion. Med. Sci. Sports Exerc. 2000, 32, 1958-1963. [CrossRef]

43. Rivers, W.H.; Webber, H.N. The action of caffeine on the capacity for muscular work. J. Physiol. 1907, 36, 33-47. [CrossRef]

44. Goldstein, E.R.; Ziegenfuss, T.; Kalman, D.; Kreider, R.; Campbell, B.; Wilborn, C.; Taylor, L.; Willoughby, D.; Stout, J.; Graves, B.S.; et al. International society of sports nutrition position stand: Caffeine and performance. J. Int. Soc. Sports Nutr. 2010, 7, 5. [CrossRef] [PubMed]

45. Killen, L.G.; Green, J.M.; O’Neal, E.K.; McIntosh, J.R.; Hornsby, J.; Coates, T.E. Effects of caffeine on session ratings of perceived exertion. Eur. J. Appl. Physiol. 2013, 113, 721-727. [CrossRef] [PubMed]

46. Vicente-Salar, N.; Santos-Sánchez, G.; Roche, E. Nutritional Ergogenic Aids in Racquet Sports: A Systematic Review. Nutrients 2020, 12, 2842. [CrossRef] [PubMed]

47. Steele, D.S.; Smith, G.L.; Miller, D.J. The effects of taurine on Ca2+ uptake by the sarcoplasmic reticulum and Ca2+ sensitivity of chemically skinned rat heart. J. Physiol. 1990, 422, 499-511. [CrossRef]

48. Stapleton, P.P.; Charles, R.P.; Redmond, H.P.; Bouchier-Hayes, D.J. Taurine and human nutrition. Clin. Nutr. 1997, 16, 103-108. [CrossRef]

49. Spriet, L.L.; Whitfield, J. Taurine and skeletal muscle function. Curr. Opin. Clin. Nutr. Metab. Care 2015, 18, 96-101. [CrossRef]

50. Hamilton, E.J.; Berg, H.M.; Easton, C.J.; Bakker, A.J. The effect of taurine depletion on the contractile properties and fatigue in fast-twitch skeletal muscle of the mouse. Amino Acids 2006, 31, 273-278. [CrossRef]

51. Huxtable, R.J. Physiological actions of taurine. Physiol. Rev. 1992, 72, 101-163. [CrossRef]

52. Balshaw, T.G.; Bampouras, T.M.; Barry, T.J.; Sparks, S.A. The effect of acute taurine ingestion on 3-km running performance in trained middle-distance runners. Amino Acids 2013, 44, 555-561. [CrossRef]

53. Rutherford, J.A.; Spriet, L.L.; Stellingwerff, T. The effect of acute taurine ingestion on endurance performance and metabolism in well-trained cyclists. Int. J. Sport Nutr. Exerc. Metab. 2010, 20, 322-329. [CrossRef]

54. Zhang, M.; Izumi, I.; Kagamimori, S.; Sokejima, S.; Yamagami, T.; Liu, Z.; Qi, B. Role of taurine supplementation to prevent exercise-induced oxidative stress in healthy young men. Amino Acids 2004, 26, 203-207. [CrossRef]

55. Waldron, M.; Patterson, S.D.; Tallent, J.; Jeffries, O. The Effects of an Oral Taurine Dose and Supplementation Period on Endurance Exercise Performance in Humans: A Meta-Analysis. Sports Med. 2018, 48, 1247-1253. [CrossRef] [PubMed]

56. Huxtable, R.; Bressler, R. Taurine concentrations in congestive heart failure. Science 1974, 184, 1187-1188. [CrossRef] [PubMed]

57. Hoyte, C.O.; Albert, D.; Heard, K.J. The use of energy drinks, dietary supplements, and prescription medications by United States college students to enhance athletic performance. J. Community Health 2013, 38, 575-580. [CrossRef]

58. Quinlivan, A.; Irwin, C.; Grant, G.D.; Anoopkumar-Dukie, S.; Skinner, T.; Leveritt, M.; Desbrow, B. The effects of Red Bull energy drink compared with caffeine on cycling time-trial performance. Int. J. Sports Physiol. Perform. 2015, 10, 897-901. [CrossRef]

59. Cureton, K.J.; Warren, G.L.; Millard-Stafford, M.L.; Wingo, J.E.; Trilk, J.; Buyckx, M. Caffeinated sports drink: Ergogenic effects and possible mechanisms. Int. J. Sport Nutr. Exerc. Metab. 2007, 17, 35-55. [CrossRef] [PubMed]

60. Ivy, J.L.; Kammer, L.; Ding, Z.; Wang, B.; Bernard, J.R.; Liao, Y.H.; Hwang, J. Improved cycling time-trial performance after ingestion of a caffeine energy drink. Int. J. Sport Nutr. Exerc. Metab. 2009, 19, 61-78. [CrossRef]

61. Kovacs, E.M.; Stegen, J.; Brouns, F. Effect of caffeinated drinks on substrate metabolism, caffeine excretion, and performance. J. Appl. Physiol. (1985) 1998, 85, 709-715. [CrossRef]

62. Del Coso, J.; Portillo, J.; Muñoz, G.; Abián-Vicén, J.; Gonzalez-Millán, C.; Muñoz-Guerra, J. Caffeine-containing energy drink improves sprint performance during an international rugby sevens competition. Amino Acids 2013, 44, 1511-1519. [CrossRef]

63. Del Coso, J.; Portillo, J.; Salinero, J.J.; Lara, B.; Abian-Vicen, J.; Areces, F. Caffeinated Energy Drinks Improve High-Speed Running in Elite Field Hockey Players. Int. J. Sport Nutr. Exerc. Metab. 2016, 26, 26-32. [CrossRef] 
64. Pérez-López, A.; Salinero, J.J.; Abian-Vicen, J.; Valadés, D.; Lara, B.; Hernandez, C.; Areces, F.; González, C.; Del Coso, J. Caffeinated energy drinks improve volleyball performance in elite female players. Med. Sci. Sports Exerc. 2015, 47, 850-856. [CrossRef] [PubMed]

65. Lara, B.; Ruiz-Vicente, D.; Areces, F.; Abián-Vicén, J.; Salinero, J.J.; Gonzalez-Millán, C.; Gallo-Salazar, C.; Del Coso, J. Acute consumption of a caffeinated energy drink enhances aspects of performance in sprint swimmers. Br. J. Nutr. 2015, 114, 908-914. [CrossRef]

66. Abian, P.; Del Coso, J.; Salinero, J.J.; Gallo-Salazar, C.; Areces, F.; Ruiz-Vicente, D.; Lara, B.; Soriano, L.; Muñoz, V.; Abian-Vicen, J. The ingestion of a caffeinated energy drink improves jump performance and activity patterns in elite badminton players. J. Sports Sci. 2015, 33, 1042-1050. [CrossRef]

67. Gallo-Salazar, C.; Areces, F.; Abián-Vicén, J.; Lara, B.; Salinero, J.J.; Gonzalez-Millán, C.; Portillo, J.; Muñoz, V.; Juarez, D.; Del Coso, J. Enhancing physical performance in elite junior tennis players with a caffeinated energy drink. Int. J. Sports Physiol. Perform. 2015, 10, 305-310. [CrossRef] [PubMed]

68. Rahnama, N.; Gaeini, A.A.; Kazemi, F. The effectiveness of two energy drinks on selected indices of maximal cardiorespiratory fitness and blood lactate levels in male athletes. J. Res. Med. Sci. 2010, 15, 127-132.

69. Ganio, M.S.; Klau, J.F.; Lee, E.C.; Yeargin, S.W.; McDermott, B.P.; Buyckx, M.; Maresh, C.M.; Armstrong, L.E. Effect of various carbohydrate-electrolyte fluids on cycling performance and maximal voluntary contraction. Int. J. Sport Nutr. Exerc. Metab. 2010, 20, 104-114. [CrossRef] [PubMed]

70. Sünram-Lea, S.I.; Owen-Lynch, J.; Robinson, S.J.; Jones, E.; Hu, H. The effect of energy drinks on cortisol levels, cognition and mood during a fire-fighting exercise. Psychopharmacology 2012, 219, 83-97. [CrossRef] [PubMed]

71. Ratamess, N.A.; Hoffman, J.R.; Ross, R.; Shanklin, M.; Faigenbaum, A.D.; Kang, J. Effects of an amino acid/creatine energy supplement on the acute hormonal response to resistance exercise. Int. J. Sport Nutr. Exerc. Metab. 2007, 17, 608-623. [CrossRef]

72. Astley, C.; Souza, D.B.; Polito, M.D. Acute Specific Effects of Caffeine-containing Energy Drink on Different Physical Performances in Resistance-trained Men. Int. J. Exerc. Sci. 2018, 11, 260-268.

73. Alford, C.; Cox, H.; Wescott, R. The effects of red bull energy drink on human performance and mood. Amino Acids 2001, 21, 139-150. [CrossRef]

74. Chtourou, H.; Trabelsi, K.; Ammar, A.; Shephard, R.J.; Bragazzi, N.L. Acute Effects of an "Energy Drink" on Short-Term Maximal Performance, Reaction Times, Psychological and Physiological Parameters: Insights from a Randomized Double-Blind, Placebo-Controlled, Counterbalanced Crossover Trial. Nutrients 2019, 11, 992. [CrossRef]

75. Jacobson, B.H.; Hester, G.M.; Palmer, T.B.; Williams, K.; Pope, Z.K.; Sellers, J.H.; Conchola, E.C.; Woolsey, C.; Estrada, C. Effect of Energy Drink Consumption on Power and Velocity of Selected Sport Performance Activities. J. Strength Cond. Res. 2018, 32, 1613-1618. [CrossRef] [PubMed]

76. Thomas, C.J.; Rothschild, J.; Earnest, C.P.; Blaisdell, A. The Effects of Energy Drink Consumption on Cognitive and Physical Performance in Elite League of Legends Players. Sports 2019, 7, 196. [CrossRef] [PubMed]

77. Umaña-Alvarado, M.; Moncada-Jiménez, J. Consumption of an "energy drink" does not improve aerobic performance in male athletes. Int. J. Appl. Sports Sci. 2005, 17, 26-34.

78. Candow, D.G.; Kleisinger, A.K.; Grenier, S.; Dorsch, K.D. Effect of sugar-free Red Bull energy drink on high-intensity run time-to-exhaustion in young adults. J. Strength Cond. Res. 2009, 23, 1271-1275. [CrossRef] [PubMed]

79. Agnol, T.D.; Souza, P.F.A.D. Efeitos fisiológicos agudos da taurina contida em uma bebida energética em indivíduos fisicamente ativos. Revista Brasileira Med. Esporte 2009, 15, 123-126. [CrossRef]

80. Kammerer, M.; Jaramillo, J.A.; García, A.; Calderón, J.C.; Valbuena, L.H. Effects of energy drink major bioactive compounds on the performance of young adults in fitness and cognitive tests: A randomized controlled trial. J. Int. Soc. Sports Nutr. 2014, 11, 44 [CrossRef] [PubMed]

81. Pettitt, R.W.; Niemeyer, J.D.; Sexton, P.J.; Lipetzky, A.; Murray, S.R. Do the noncaffeine ingredients of energy drinks affect metabolic responses to heavy exercise? J. Strength Cond. Res. 2013, 27, 1994-1999. [CrossRef]

82. Nelson, M.T.; Biltz, G.R.; Dengel, D.R. Cardiovascular and ride time-to-exhaustion effects of an energy drink. J. Int. Soc. Sports Nutr. 2014, 11, 2. [CrossRef]

83. Hoffman, J.R.; Kang, J.; Ratamess, N.A.; Hoffman, M.W.; Tranchina, C.P.; Faigenbaum, A.D. Examination of a pre-exercise, high energy supplement on exercise performance. J. Int. Soc. Sports Nutr. 2009, 6, 2. [CrossRef]

84. Eckerson, J.M.; Bull, A.J.; Baechle, T.R.; Fischer, C.A.; O’Brien, D.C.; Moore, G.A.; Yee, J.C.; Pulverenti, T.S. Acute ingestion of sugar-free red bull energy drink has no effect on upper body strength and muscular endurance in resistance trained men. $J$. Strength Cond. Res. 2013, 27, 2248-2254. [CrossRef]

85. Goel, V.; Manjunatha, S.; Pai, K.M. Effect of red bull energy drink on auditory reaction time and maximal voluntary contraction. Indian J. Physiol. Pharmacol. 2014, 58, 17-21.

86. Cappelletti, S.; Piacentino, D.; Sani, G.; Aromatario, M. Caffeine: Cognitive and physical performance enhancer or psychoactive drug? Curr. Neuropharmacol. 2015, 13, 71-88. [CrossRef] [PubMed]

87. Reissig, C.J.; Strain, E.C.; Griffiths, R.R. Caffeinated energy drinks-A growing problem. Drug Alcohol Depend. 2009, 99, 1-10. [CrossRef] [PubMed]

88. Petit, A.; Levy, F.; Lejoyeux, M.; Reynaud, M.; Karila, L. Energy drinks: An unknown risk. Rev. Prat. 2012, 62, 673-678. [PubMed] 
89. Holmgren, P.; Nordén-Pettersson, L.; Ahlner, J. Caffeine fatalities—four case reports. Forensic Sci. Int. 2004, 139, 71-73. [CrossRef] [PubMed]

90. Kerrigan, S.; Lindsey, T. Fatal caffeine overdose: Two case reports. Forensic Sci. Int. 2005, 153, 67-69. [CrossRef] [PubMed]

91. Rudolph, T.; Knudsen, K. A case of fatal caffeine poisoning. Acta Anaesthesiol. Scand. 2010, 54, 521-523. [CrossRef] [PubMed]

92. Sepkowitz, K.A. Energy drinks and caffeine-related adverse effects. JAMA 2013, 309, 243-244. [CrossRef] [PubMed]

93. Nawrot, P.; Jordan, S.; Eastwood, J.; Rotstein, J.; Hugenholtz, A.; Feeley, M. Effects of caffeine on human health. Food Addit. Contam. 2003, 20, 1-30. [CrossRef] [PubMed]

94. Dhar, R.; Stout, C.W.; Link, M.S.; Homoud, M.K.; Weinstock, J.; Estes, N.A., 3rd. Cardiovascular toxicities of performanceenhancing substances in sports. Mayo Clin. Proc. 2005, 80, 1307-1315. [CrossRef]

95. Shekelle, P.G.; Hardy, M.L.; Morton, S.C.; Maglione, M.; Mojica, W.A.; Suttorp, M.J.; Rhodes, S.L.; Jungvig, L.; Gagné, J. Efficacy and safety of ephedra and ephedrine for weight loss and athletic performance: A meta-analysis. JAMA 2003, 289, 1537-1545. [CrossRef] [PubMed]

96. Williams, A.D.; Cribb, P.J.; Cooke, M.B.; Hayes, A. The effect of ephedra and caffeine on maximal strength and power in resistance-trained athletes. J. Strength Cond. Res. 2008, 22, 464-470. [CrossRef]

97. Haller, C.A.; Jacob, P., 3rd; Benowitz, N.L. Enhanced stimulant and metabolic effects of combined ephedrine and caffeine. Clin. Pharmacol. Ther. 2004, 75, 259-273. [CrossRef]

98. Fenster, L.; Quale, C.; Hiatt, R.A.; Wilson, M.; Windham, G.C.; Benowitz, N.L. Rate of caffeine metabolism and risk of spontaneous abortion. Am. J. Epidemiol. 1998, 147, 503-510. [CrossRef]

99. Rétey, J.V.; Adam, M.; Honegger, E.; Khatami, R.; Luhmann, U.F.; Jung, H.H.; Berger, W.; Landolt, H.P. A functional genetic variation of adenosine deaminase affects the duration and intensity of deep sleep in humans. Proc. Natl. Acad. Sci. USA 2005, 102, 15676-15681. [CrossRef]

100. Rétey, J.V.; Adam, M.; Khatami, R.; Luhmann, U.F.; Jung, H.H.; Berger, W.; Landolt, H.P. A genetic variation in the adenosine A2A receptor gene (ADORA2A) contributes to individual sensitivity to caffeine effects on sleep. Clin. Pharmacol. Ther. 2007, 81, 692-698. [CrossRef]

101. Rogers, P.J.; Hohoff, C.; Heatherley, S.V.; Mullings, E.L.; Maxfield, P.J.; Evershed, R.P.; Deckert, J.; Nutt, D.J. Association of the anxiogenic and alerting effects of caffeine with ADORA2A and ADORA1 polymorphisms and habitual level of caffeine consumption. Neuropsychopharmacology 2010, 35, 1973-1983. [CrossRef] [PubMed]

102. Erblang, M.; Drogou, C.; Gomez-Merino, D.; Metlaine, A.; Boland, A.; Deleuze, J.F.; Thomas, C.; Sauvet, F.; Chennaoui, M. The Impact of Genetic Variations in ADORA2A in the Association between Caffeine Consumption and Sleep. Genes 2019, $10,1021$. [CrossRef] [PubMed]

103. Iio, W.; Matsukawa, N.; Tsukahara, T.; Toyoda, A. The effects of oral taurine administration on behavior and hippocampal signal transduction in rats. Amino Acids 2012, 43, 2037-2046. [CrossRef]

104. Horvath, D.M.; Murphy, R.M.; Mollica, J.P.; Hayes, A.; Goodman, C.A. The effect of taurine and $\beta$-alanine supplementation on taurine transporter protein and fatigue resistance in skeletal muscle from mdx mice. Amino Acids 2016, 48, 2635-2645. [CrossRef]

105. Womack, C.J.; Saunders, M.J.; Bechtel, M.K.; Bolton, D.J.; Martin, M.; Luden, N.D.; Dunham, W.; Hancock, M. The influence of a CYP1A2 polymorphism on the ergogenic effects of caffeine. J. Int. Soc. Sports Nutr. 2012, 9, 7. [CrossRef] [PubMed]

106. Salinero, J.J.; Lara, B.; Ruiz-Vicente, D.; Areces, F.; Puente-Torres, C.; Gallo-Salazar, C.; Pascual, T.; Del Coso, J. CYP1A2 Genotype Variations Do Not Modify the Benefits and Drawbacks of Caffeine during Exercise: A Pilot Study. Nutrients 2017, 9, 269. [CrossRef]

107. Pataky, M.W.; Womack, C.J.; Saunders, M.J.; Goffe, J.L.; D’Lugos, A.C.; El-Sohemy, A.; Luden, N.D. Caffeine and 3-km cycling performance: Effects of mouth rinsing, genotype, and time of day. Scand. J. Med. Sci. Sports 2016, 26, 613-619. [CrossRef]

108. Carswell, A.T.; Howland, K.; Martinez-Gonzalez, B.; Baron, P.; Davison, G. The effect of caffeine on cognitive performance is influenced by CYP1A2 but not ADORA2A genotype, yet neither genotype affects exercise performance in healthy adults. Eur. J. Appl. Physiol. 2020, 120, 1495-1508. [CrossRef] [PubMed]

109. Spineli, H.; Pinto, M.P.; Dos Santos, B.P.; Lima-Silva, A.E.; Bertuzzi, R.; Gitaí, D.L.G.; de Araujo, G.G. Caffeine improves various aspects of athletic performance in adolescents independent of their 163 C > A CYP1A2 genotypes. Scand. J. Med. Sci. Sports 2020. [CrossRef] [PubMed]

110. Glaister, M.; Chopra, K.; Pereira De Sena, A.; Sternbach, C.; Morina, L.; Mavrommatis, Y. Caffeine, exercise physiology, and time-trial performance: No effect of ADORA2A or CYP1A2 genotypes. Appl. Physiol. Nutr. Metab. 2020. [CrossRef]

111. Davenport, A.D.; Jameson, T.S.O.; Kilroe, S.P.; Monteyne, A.J.; Pavis, G.F.; Wall, B.T.; Dirks, M.L.; Alamdari, N.; Mikus, C.R.; Stephens, F.B. A Randomised, Placebo-Controlled, Crossover Study Investigating the Optimal Timing of a Caffeine-Containing Supplement for Exercise Performance. Sports Med. Open 2020, 6, 17. [CrossRef] [PubMed]

112. Higgins, J.P.; Tuttle, T.D.; Higgins, C.L. Energy beverages: Content and safety. Mayo Clin. Proc. 2010, 85, 1033-1041. [CrossRef]

113. Benowitz, N.L. Clinical pharmacology of caffeine. Annu Rev. Med. 1990, 41, 277-288. [CrossRef]

114. Davis, J.M.; Zhao, Z.; Stock, H.S.; Mehl, K.A.; Buggy, J.; Hand, G.A. Central nervous system effects of caffeine and adenosine on fatigue. Am. J. Physiol. Regul. Integr. Comp. Physiol. 2003, 284, R399-R404. [CrossRef] [PubMed]

115. Bazzucchi, I.; Felici, F.; Montini, M.; Figura, F.; Sacchetti, M. Caffeine improves neuromuscular function during maximal dynamic exercise. Muscle Nerve 2011, 43, 839-844. [CrossRef] [PubMed]

116. McPherson, P.S.; Kim, Y.K.; Valdivia, H.; Knudson, C.M.; Takekura, H.; Franzini-Armstrong, C.; Coronado, R.; Campbell, K.P. The brain ryanodine receptor: A caffeine-sensitive calcium release channel. Neuron 1991, 7, 17-25. [CrossRef] 
117. Van Soeren, M.H.; Graham, T.E. Effect of caffeine on metabolism, exercise endurance, and catecholamine responses after withdrawal. J. Appl. Physiol. (1985) 1998, 85, 1493-1501. [CrossRef]

118. Gutiérrez-Hellín, J.; Del Coso, J. Effects of p-Synephrine and Caffeine Ingestion on Substrate Oxidation during Exercise. Med. Sci. Sports Exerc. 2018, 50, 1899-1906. [CrossRef]

119. Portillo, J.; Del Coso, J.; Abián-Vicén, J. Effects of Caffeine Ingestion on Skill Performance During an International Female Rugby Sevens Competition. J. Strength Cond. Res. 2017, 31, 3351-3357. [CrossRef]

120. Watson, E.J.; Coates, A.M.; Kohler, M.; Banks, S. Caffeine Consumption and Sleep Quality in Australian Adults. Nutrients $2016,8,479$. [CrossRef]

121. Curran, C.P.; Marczinski, C.A. Taurine, caffeine, and energy drinks: Reviewing the risks to the adolescent brain. Birth Defects Res. 2017, 109, 1640-1648. [CrossRef] [PubMed]

122. Pickering, C.; Kiely, J. What Should We Do About Habitual Caffeine Use in Athletes? Sports Med. 2019, 49, 833-842. [CrossRef]

123. Pallarés, J.G.; Fernández-Elías, V.E.; Ortega, J.F.; Muñoz, G.; Muñoz-Guerra, J.; Mora-Rodríguez, R. Neuromuscular responses to incremental caffeine doses: Performance and side effects. Med. Sci. Sports Exerc. 2013, 45, 2184-2192. [CrossRef]

124. Nowak, D.; Gośliński, M.; Nowatkowska, K. The Effect of Acute Consumption of Energy Drinks on Blood Pressure, Heart Rate and Blood Glucose in the Group of Young Adults. Int. J. Environ. Res. Public Health 2018, 15, 544. [CrossRef] [PubMed]

125. Hajsadeghi, S.; Mohammadpour, F.; Manteghi, M.J.; Kordshakeri, K.; Tokazebani, M.; Rahmani, E.; Hassanzadeh, M. Effects of energy drinks on blood pressure, heart rate, and electrocardiographic parameters: An experimental study on healthy young adults. Anatol. J. Cardiol. 2016, 16, 94-99. [CrossRef]

126. Miles-Chan, J.L.; Charrière, N.; Grasser, E.K.; Montani, J.P.; Dulloo, A.G. The blood pressure-elevating effect of Red Bull energy drink is mimicked by caffeine but through different hemodynamic pathways. Physiol. Rep. 2015, 3. [CrossRef]

127. Grgic, J.; Pickering, C.; Bishop, D.J.; Del Coso, J.; Schoenfeld, B.J.; Tinsley, G.M.; Pedisic, Z. ADOR2A C Allele Carriers Exhibit Ergogenic Responses to Caffeine Supplementation. Nutrients 2020, 12, 741. [CrossRef] [PubMed]

128. Martins, G.L.; Guilherme, J.; Ferreira, L.H.B.; de Souza-Junior, T.P.; Lancha, A.H., Jr. Caffeine and Exercise Performance: Possible Directions for Definitive Findings. Front. Sports Act. Living 2020, 2, 574854. [CrossRef]

129. Pickering, C. Caffeine, CYP1A2 genotype, and sports performance: Is timing important? Ir. J. Med. Sci. 2019, 188, 349-350. [CrossRef] [PubMed]

130. Bell, D.G.; McLellan, T.M. Exercise endurance 1, 3, and $6 \mathrm{~h}$ after caffeine ingestion in caffeine users and nonusers. J. Appl. Physiol. (1985) 2002, 93, 1227-1234. [CrossRef] [PubMed]

131. Ahmetov, I.I.; Egorova, E.S.; Gabdrakhmanova, L.J.; Fedotovskaya, O.N. Genes and Athletic Performance: An Update. Med. Sport Sci. 2016, 61, 41-54. [CrossRef] [PubMed]

132. Joyner, M.J. Genetic Approaches for Sports Performance: How Far Away Are We? Sports Med. 2019. [CrossRef] [PubMed]

133. Varillas Delgado, D.; Telleria Orriols, J.J.; Martin Saborido, C. Liver-Metabolizing Genes and Their Relationship to the Performance of Elite Spanish Male Endurance Athletes; a Prospective Transversal Study. Sports Med. Open 2019, 5, 50. [CrossRef] [PubMed] 